\title{
WILEY-VCH
}

\section{Stability and Dark Hysteresis Correlate in NiO-based Perovskite Solar Cells}

Diego Di Girolamo, Fabio Matteocci, Felix Utama Kosasih, Ganna Chistiakova, Weiwei Zuo,

Giorgio Divitini, Lars Korte, Caterina Ducati, Aldo Di Carlo, Danilo Dini, Antonio Abate*

Diego Di Girolamo, Prof. Danilo Dini

Department of Chemistry

University of Rome La Sapienza

Piazzale Aldo Moro 5, Rome, 00185, Italy

Dr. Fabio Matteocci, Prof. Aldo Di Carlo

C.H.O.S.E - Centre for Hybrid and Organic Solar Energy, Department of Electronic Engineering University of Rome Tor Vergata

Via del politecnico 1, Rome, 00133, Italy

Prof. Aldo Di Carlo

L.A.S.E - Laboratory of Advanced Solar Energy

National University of Science and Technology NUST-MISiS

119049 Leninskiy prospect 6, Moscow, Russia

Felix Utama Kosasih, Dr. Giorgio Divitini, Prof. Caterina Ducati

Department of Materials Science and Metallurgy

University of Cambridge

27 Charles Babbage Road, Cambridge CB3 0FS, United Kingdom

Dr. Antonio Abate, Ganna Chistiakova, Dr. Lars Korte

Institute for Silicon Photovoltaics

Helmholtz-Zentrum Berlin für Materialien und Energie GmbH

Kekuléstraße 5, D-12489 Berlin, Germany

mail: antonio.abate@helmholtz-berlin.de

Dr. Antonio Abate

State Key Laboratory of Photocatalysis on Energy and Environment Institute of Advanced Energy

Materials

Fuzhou University

Fuzhou, Fujian 350002, China

and

Department od Chemical, Materials and Production Engineering

University of Naples Federico II

Piazzale Tecchio 80, 80125 Fuorigrotta, Naples, Italy

Keywords: Perovskite Solar Cells, Hysteresis, Stability, Interface Engineering, NiO 


\title{
WILEY-VCH
}

\begin{abstract}
In perovskite solar cell, the interfaces are a weak link with respect to degradation. Electrochemical reactivity of the perovskite's halides has been reported for both molecular and polymeric hole selective layers (HSLs), and here we show that also NiO brings about this decomposition mechanism. Employing NiO as a HSL in p-i-n PSCs with PCE of 16.8\%, we found non-capacitive hysteresis in the dark, which is attributable to the bias-induced degradation of perovskite/NiO interface. We explored the possibility of electrochemically decoupling $\mathrm{NiO}$ from the perovskite via the introduction of a buffer layer. Employing a hybrid magnesium-organic interlayer, we entirely suppressed the noncapacitive hysteresis and improved the device's electrical stability. At the same time, the PCE is improved up to $18 \%$ thanks to reduced interfacial charge recombination, which enables more efficient hole collection resulting in higher Voc and FF.
\end{abstract}




\section{WILEY-VCH}

\section{Introduction}

Halide perovskite solar cells (PSCs) revolutionised photovoltaics in the last decade, primarily due to an outstanding power conversion efficiency (PCE), with the certified record at $24.2 \%[1]$. Unfortunately, this high PCE remains ephemeral given the severe instability suffered by PSCs. PSC lifetimes in literature are still far from the goal of 25 years needed for commercialization[2,3]. Thus, if metal halide perovskite (PSK) solar cells are to take part in supplying the world's energy demand, device stability must be significantly improved.

Several kinds of stimuli have been found to degrade PSCs. When exposed to moist atmosphere, $\mathrm{CH}_{3} \mathrm{NH}_{3} \mathrm{PbI}_{3}$ (MAPI) undergoes an irreversible degradation via the formation of hydrated phases.[4,5] Even the all-inorganic perovskite $\mathrm{CsPbBr}_{3}$ undergoes intricate structural modifications when exposed to relative humidity $(\mathrm{RH})$ above $60 \%$, highlighting the sensitivity of lead halide perovskites to moisture also in the absence of organic cations[6]. Perovskites are also sensitive to atmospheric oxygen, which can rapidly diffuse inside the perovskite film through grain boundaries and inside the lattice via iodine vacancies[7]. Under illumination, the superoxide anion $\left(\mathrm{O}_{2}^{-}\right)$forms, which is highly reactive and triggers lattice decomposition[8,9].

Nevertheless, proper encapsulation procedures[10,11] can mitigate or, in the best scenario, eliminate the influence of moisture or oxygen on device stability. In addition to that, metal halide perovskites are also sensitive to heat, illumination and electrical bias, three certain conditions in photovoltaic operation. A detailed understanding of thermal and photoelectrical failure mechanisms is one of the most critical topics that must be addressed.[3] Thermal instability is mainly associated with methylammonium decomposition to volatile methylamine[12]. Moreover, ionic diffusion from electrodes is enhanced at high temperatures and causes irreversible degradation[13-15]. As far as photo-instability is concerned, the photo-induced segregation of halides in multi-halide perovskites represents an important microscopic factor at the basis of the lack of photostability in these systems[16,17]. Furthermore, UV light accelerates perovskite degradation, for instance, by promoting the reaction of perovskites with $\mathrm{TiO} 2[18]$, and photo-oxidation of iodide to interstitial iodine[19]. 


\section{WILEY-VCH}

Interestingly, together with the decomposition paths it brings about, the iodide photoelectrochemistry has been recently invoked as a possible reason for the high defect tolerance of perovskites[20].

In the following paragraphs, we will focus on the effects caused by electrical bias. Since charge carrier transport in perovskites is governed by mixed electronic-ionic conduction, halide perovskites undergo structural modification under the influence of an electric field[21-23]. This mixedconduction nature affects short- and long-term photovoltaic performance: hysteresis[24,25], and degradation/recovery upon light/dark cycling[2,26]. These two phenomena are directly related. Intrinsic properties of perovskites alone cannot explain all the hysteretic phenomenology reported to date, with the best example being the fact that p-i-n PSCs are nearly unaffected by hysteresis, even if the perovskite stoichiometry is the same as that used in the analogous n-i-p configuration. The physicochemical processes at the interface between perovskite and selective contacts must be considered. Substantial efforts were directed to the characterisation and investigation of ionic migration and diffusion across interfaces, especially when selective contact materials are molecular or polymeric[27]. Atoms and ions from the metallic electrode or the transparent conductive oxide can spread through the device as well[13,28]. In the case of inorganic selective contacts, ionic migration across interfaces is severely hindered, but the interface cannot be considered inert. De Angelis[29] and Garcia-Belmonte[30] described the Ti-I-Pb bond forming at the perovskite/ $\mathrm{TiO}_{2}$ interface. Similarly, Rand and co-workers pointed out the sensitivity of $\mathrm{CH}_{3} \mathrm{NH}_{3} \mathrm{PbI}_{3}$ and $\mathrm{TiO}_{2}$ photoelectrical properties to iodine and oxygen exposure[31] speculating on the possible effect in photovoltaic devices. Both $\mathrm{TiO}_{2}$ and $\mathrm{SnO}_{2}$ are reported to exchange ions (oxygen or hydrogen) with perovskites[32,33], which likely is a common feature of metal-oxide / perovskite interfaces. The chemistry at perovskite / selective contact interfaces is not limited to absorption/desorption equilibria. Garcia-Belmonte observed an electrochemical reaction between spiro-OMeTAD and iodide, which also occurs with PEDOT:PSS[34]. From these critical contributions, two main concepts arise: oxide 


\section{WILEY-VCH}

semiconductors generally interact with perovskites, and the interface of perovskite with a p-type semiconductor can be the site of unwanted redox reactions.

In this work, we focused our attention on the $\mathrm{NiO} /$ perovskite interface in $\mathrm{p}$-i-n PSCs. $\mathrm{NiO}$ is a p-type oxide, and potentially presents the two criticalities just discussed, yet it is a promising choice in terms of stability[35]. Measuring J-V curves on these cells in the dark, we observed what is referred to as non-capacitive hysteresis, which has been suggested to denote a degradation proceess[34]. The dual aim of this work is, first, to provide new insights into the interface instability and, second, to investigate strategies to overcome it. The engineering of the $\mathrm{NiO} /$ perovskites interface has been already successfully employed to obtain a perovskite film of better quality[36] and to improve the hole transfer[37], together with the suppression of interface recombination[38], and the enhancement of the device photostability[39]. Therefore, it appears as a promising strategy also to avoid detrimental interaction between $\mathrm{NiO}$ and perovskites under the effect of the electrical bias. Here, by exploring different buffer layers to eliminate the non-capacitive hysteresis, we developed a hybrid $\mathrm{Mg}^{2+}$ based interlayer as a potential solution. This interlayer reduces recombination at the $\mathrm{NiO} /$ perovskite interface, resulting in a higher PCE. More interestingly, the maximum power point tracking (MPPT) outputs were stabilised. This stability toward electrical bias is further demonstrated by measuring the MPPT after a severe polarisation in dark conditions at potentials above $1 \mathrm{~V}$, which again yielded a stable power output. Our results directly link the non-capacitive hysteresis to device degradation and show that addressing the interface reactivity is an important route to improve perovskite photovoltaics. 


\section{WILEY-VCH}

\section{Results and Discussion}

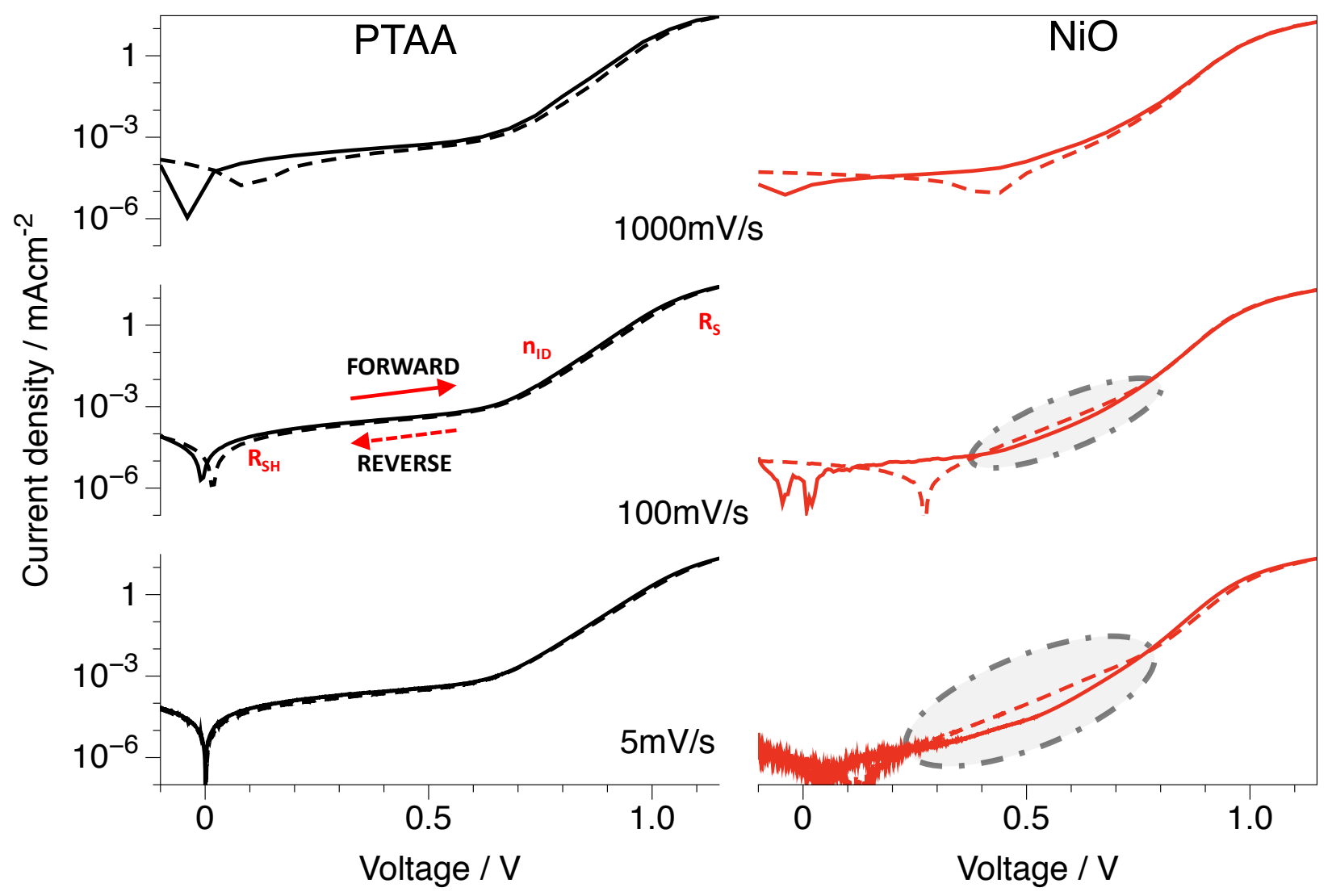

Figure 1. The left column reports JV curves recorded in the dark for PTAA-based PSCs at different scan rates: 1000 $\mathrm{mV} / \mathrm{s}, 100 \mathrm{mV} / \mathrm{s}$ and $5 \mathrm{mV} / \mathrm{s}$ from top to bottom. The shunt $\left(\mathrm{RsH}_{\mathrm{sH}}\right)$ and series ( $\mathrm{Rs}_{\text {s }}$ resistance regimes, together with the legend for scan direction (forward is from $-0.1 \mathrm{~V}$ to $1.2 \mathrm{~V}$ ) are marked on the $100 \mathrm{mV} / \mathrm{s}$ curve. The right column shows dark JV curves with $\mathrm{NiO}$ as the HSL. A hysteresis loop, increasing at lower scan rates, is observed only for NiO based devices. It is referred to as non-capacitive hysteresis in the text.

The critical observation behind this work is the hysteresis in the dark current observed for $\mathrm{NiO} /$ CsFAMA / $\mathrm{C}_{60}$ based devices, attributable to the perovskite-NiO interface since this hysteresis was not found in devices employing the organic polymer poly-[bis(4-phenyl)(2,4,6trimethylphenyl)amine] (PTAA) as the hole selective layer (HSL), which we take as a reference in Figure 1. From these JV curves, the shunt resistance $\left(\mathrm{R}_{\mathrm{SH}}\right)$ regime $(\mathrm{V}<0.5 \mathrm{~V})$ and the series resistance $\left(\mathrm{R}_{\mathrm{S}}\right)$ regime $(\mathrm{V}>1 \mathrm{~V})$ are distinguishable from the diode-like current, where the slope in the semilogarithmic plot gives the diode ideality factor $\left(\mathrm{n}_{\mathrm{id}}\right)$. Furthermore, the reverse dark current scan (from $1.2 \mathrm{~V}$ to $-0.1 \mathrm{~V}$ ) is always below the forward scan (from $-0.1 \mathrm{~V}$ to $1.2 \mathrm{~V}$ ) due to the interplay 


\section{WILEY-VCH}

between the scan rate and device capacitance[40], i.e. the capacitive hysteresis. This type of hysteresis can be reduced by slowing the scan, as observable for PTAA at $5 \mathrm{mV} / \mathrm{s}$ scan rate where hysteresis almost vanished. The dark JV loop markedly differs when the HSL is NiO. The crucial difference is that the reverse dark current is higher than the forward one around $0.5 \mathrm{~V}$ (highlighted in grey in Figure 1). A capacitive mechanism cannot explain this effect and, moreover, this hysteretic behaviour worsened at lower scan rates, when the cell is polarised for a longer time. This behaviour suggests that hysteresis is triggered by forward bias. Thus, the driving force has to be found in the potentialdependent interaction of the HSL with the perovskite film. Garcia-Belmonte and co-workers already reported a similar behaviour for n-i-p and p-i-n PSCs[34,41,42] (they coined the "non-capacitive hysteresis" term adopted herein). By focusing on the $\mathrm{TiO}_{2} / \mathrm{CH}_{3} \mathrm{NH}_{3} \mathrm{PbI}_{3} /$ spiro-OMeTAD configuration, they pointed to the p-type interface as the source of non-capacitive hysteresis, in agreement with our observations. Furthermore, they identified an electrochemical reaction between spiro-OMeTAD and iodide from $\mathrm{CH}_{3} \mathrm{NH}_{3} \mathrm{PbI}_{3}$ as the cause. In this work, we employed a mixedcation mixed-anion (triple-cation, CsFAMA)[43] perovskite composition. Nonetheless, taking into account the respective Nernst potentials, the electrochemically active ion is the iodide, as in the case of $\mathrm{CH}_{3} \mathrm{NH}_{3} \mathrm{PbI}_{3}$. The oxidation of bromide requires a higher potential $\left(\mathrm{E}^{0}{ }_{\mathrm{Br}}=1.07 \mathrm{~V}\right.$ vs $\mathrm{SHE}, \mathrm{E}_{\mathrm{I}}^{0}=$ $0.53 \mathrm{~V}$ vs SHE) and both caesium and formamidinium are supposed to be electrochemically stable in the potential range investigated, relevant for PV operation. However, the mechanism suggested for the spiro-OMeTAD / $\mathrm{CH}_{3} \mathrm{NH}_{3} \mathrm{PbI}_{3}$ interface, with the formation of a neutral spiro-iodide[44] complex followed by its diffusion away from the interface, cannot apply to our case since iodide is too large to penetrate the $\mathrm{NiO}$ lattice. 
WILEY-VCH

a
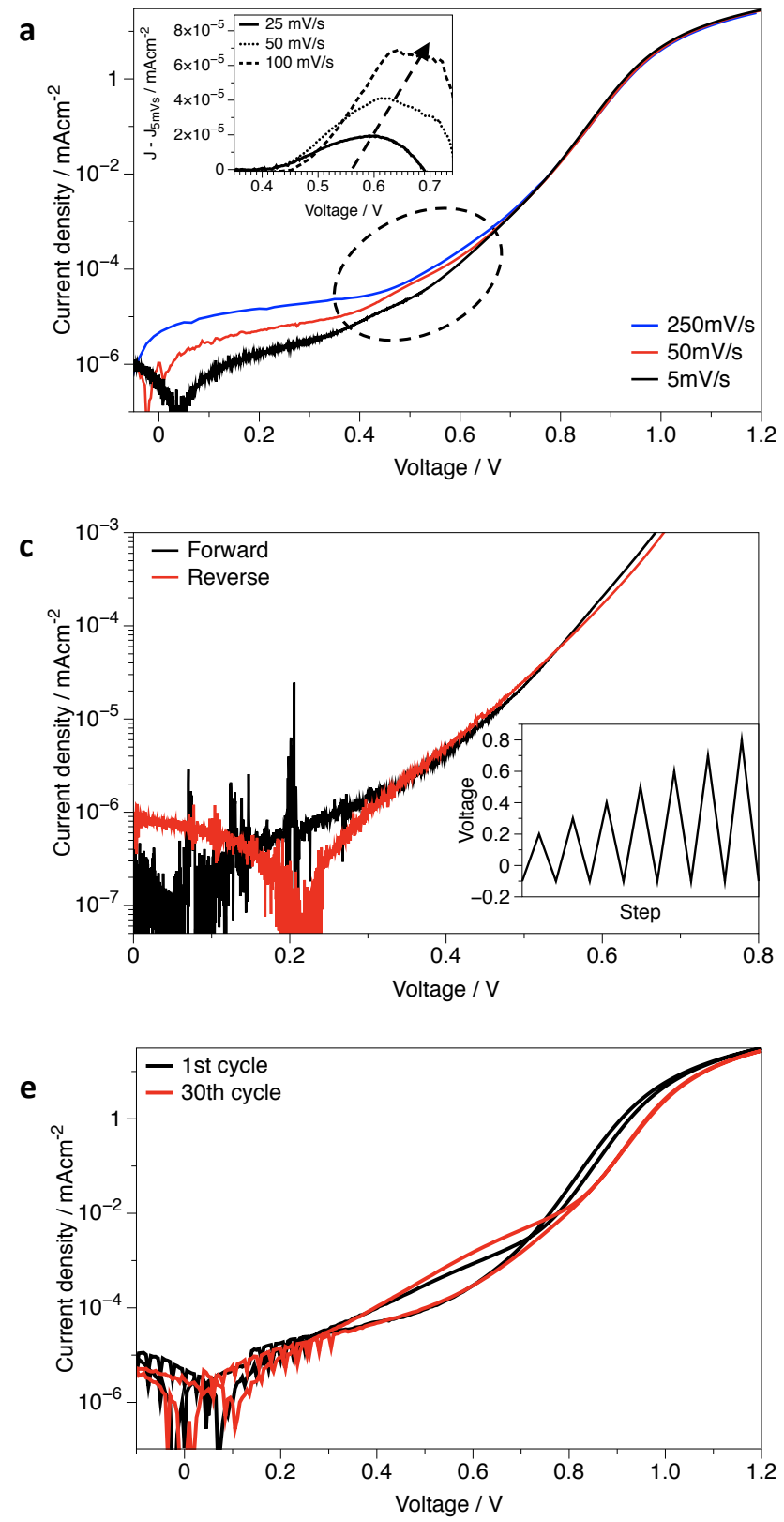

b

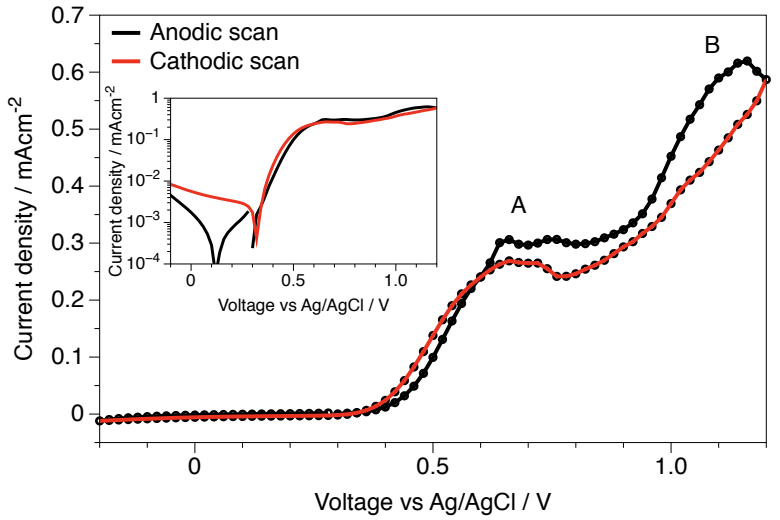

d
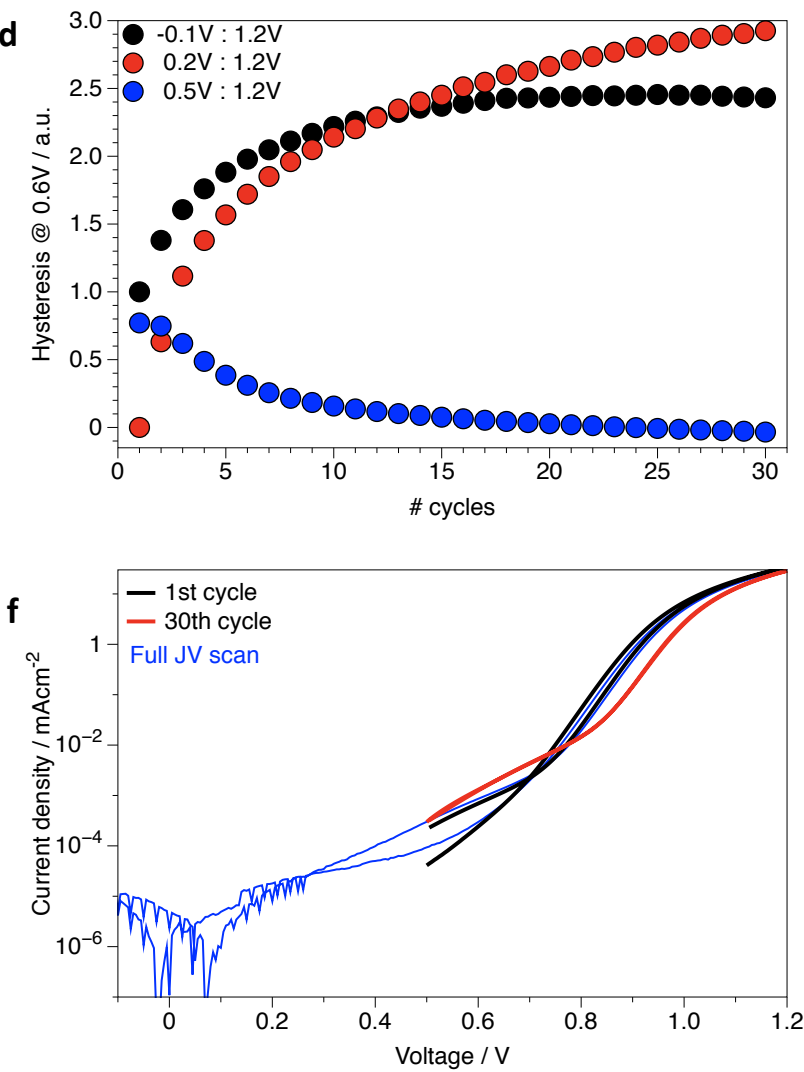

Figure 2. a) A focus on the forward scan JV curves in the dark at different scan rates measured for NiO based devices. The marked region highlights the distortion around $0.4-0.7 \mathrm{~V}$. In the inset, we report the value of $\mathrm{J}_{\mathrm{SCAN}-\mathrm{RATE}}-\mathrm{J}_{5 \mathrm{MV} / \mathrm{S}}$ for the voltage range of interest to subtract the diode current. In this way, the distortion is highlighted and appears as a peak. b) Cyclic voltammetry obtained employing ITO/NiO as WE, a Pt wire as $\mathrm{CE}$, and $\mathrm{Ag} / \mathrm{AgCl}$ as $\mathrm{RE}$ with an $\mathrm{ACN}$ electrolyte containing $\mathrm{NaI}(5 \mathrm{mM})$ and TBABF4 $(0.1 \mathrm{M})$ as supporting electrolyte. A hysteresis loop associated with iodide oxidation on $\mathrm{NiO}$ is found. This trend is shown in the semilogarithmic plot in the inset. c) The voltage scan between -0.1 and $0.8 \mathrm{~V}$ at $5 \mathrm{mV} / \mathrm{s}$ being the last step of the potential wave in the inset and showing the first appearance of the hysteresis loop. d) The evolution upon cycling of the hysteresis loop (as evaluated from the integration of the area inside the circle around $0.6 \mathrm{~V}$ ). When cycling between $0.5 \mathrm{~V}$ and $1.2 \mathrm{~V}$, the hysteresis vanished, while it stabilised in the other conditions. e) $1^{\text {st }}$ 


\section{WILEY-VCH}

and $30^{\text {th }} \mathrm{JV}$ cycles when scanning the potential between $-0.1 \mathrm{~V}$ and $1.2 \mathrm{~V}$. The hysteresis loop was increased. $\left.\mathbf{f}\right) 1^{\mathrm{st}}$ and $30^{\text {th }} \mathrm{JV}$ cycles when examining the potential between $0.5 \mathrm{~V}$ and $1.2 \mathrm{~V}$. The hysteresis loop disappeared. The full JV curves are reported for comparison.

It is interesting that, in the seminal works of Garcia-Belmonte group, the faradic peak attributed to the oxidation of perovskite at HSL interface was observed in the reverse scan in the dark[30,34]. In electrochemical experiments, the oxidation current is generally higher in the anodic scan, which here corresponds to the forward scan. In fact, in our system, we identified a peak between $0.4 \mathrm{~V}$ and $0.7 \mathrm{~V}$ in the forward scan (Figure 2a). This peak is highlighted in the inset, where we subtracted the forward scan recorded at $5 \mathrm{mV} / \mathrm{s}$ (where the peak is smaller) from the faster scans to eliminate the diode-like current. The resulting differential peak increases in magnitude and shifts toward higher potentials at faster scan rates, as expected from an irreversible electrochemical reaction[45]. Faradic current densities below $10^{-2} \mathrm{~mA} / \mathrm{cm}^{2}$ (the upper limit to the current density from the hysteresis loop as observed in the slow scan of figure 1) are compatible with reactions involving one or few atomic layers at the interface. However, we cannot rule out ionic-to-electronic current amplification[46], i.e. the modification of electronic current due to ionic processes. To gain more insight into the electrochemistry of the system, we conducted electrochemical cyclic voltammetry employing an iodide-containing electrolyte and ITO/NiO as working electrode, reported in Figures $2 \mathrm{~b}$ and $\mathrm{S} 2$. The oxidation of iodide on a $\mathrm{NiO}$ surface is a multi-step, electrochemically irreversible reaction. Two peaks characterise it, which we indicated as A and B in the voltammogram of Figure $2 \mathrm{~b}$. These are generally attributed to[47]:

$$
\begin{aligned}
& 3 \mathrm{I}^{-} \rightarrow \mathrm{I}_{3}^{-}+2 \mathrm{e} \\
& 2 \mathrm{I}_{3}^{-} \rightarrow 3 \mathrm{I}_{2}+2 \mathrm{e}
\end{aligned}
$$

We remark that the fast kinetics of those reactions on the $\mathrm{NiO}$ surface is among the limiting factors of p-type dye-sensitised solar cells[48], and the lack of a cathodic peak in the potential window investigated is in agreement with the Gerischer model of semiconductor electrochemistry[49]. More 


\section{WILEY-VCH}

interestingly, we observed a hysteresis loop in the potential range between 0.4 and $0.6 \mathrm{~V}$ vs $\mathrm{Ag} / \mathrm{AgCl}$.

The appearance of an electrochemical hysteresis loop is due to a reaction with a complex mechanism (not a simple charge transfer event), and it is typical of electrodeposition[50] and corrosion[51], i.e. processes with activation energy where the electrode surface is structurally modified. Briefly, the current in the cathodic scan is higher around the reaction onset potential due to electrode activation in the previous anodic scan.

We postulate that a similar argument applies to the NiO/CsFAMA interface. In this case, the increase in the reactivity of $\mathrm{NiO} / \mathrm{CsFAMA}$ interface with forward bias can be attributed either to electrode activation (kinetic effect) or to iodide migration towards $\mathrm{NiO}$ in forward bias (mass effect)[34]. The two effects probably cooperate. In fact, by applying a bias waveform consisting of a sequence of triangular pulses with increasing amplitudes at $5 \mathrm{mV} / \mathrm{s}$ (inset Figure 2c), starting from $0.1 \mathrm{~V}$ we had to cycle up to $0.8 \mathrm{~V}$ to detect the loop, as shown in Figures $2 \mathrm{c}$ and $\mathrm{S} 3$. This potential value is at least $0.5 \mathrm{~V}$ higher than the reaction onset (which is below $0.3 \mathrm{~V}$, see Figure S4) but is slightly lower than the cell built-in potential[52,53]. Therefore a substantial iodide accumulation cannot occur (see Note S5 for a detailed explanation).

Further, we show in Figures $2 \mathrm{~d}-\mathrm{f}$ that the electrode activation was not stable and quickly relaxed. In fact, upon cycling at $50 \mathrm{mV} / \mathrm{s}$ in the full potential range $(-0.1-1.2 \mathrm{~V}$, Figure $2 \mathrm{e})$, the hysteresis loop was observed at every scan and even increased, as if the electrode reactivity of the first forward scan was restored at each cycle. The same trend was followed by cycling in the range $0.2 \mathrm{~V}-1.2 \mathrm{~V}$. When cycling between $0.5 \mathrm{~V}$ and $1.2 \mathrm{~V}$ the hysteresis vanished (Figure $2 \mathrm{f}$ ), meaning that the relaxation process is too slow at $0.5 \mathrm{~V}$ to restore the pristine state. The relaxation was not iodine reduction (which would require a high negative overvoltage on $\mathrm{NiO}$ ); otherwise, it would not occur above $0.2 \mathrm{~V}$ (the iodide oxidation onset). A possible alternative is the diffusion/desorption of iodine away from the $\mathrm{NiO}$ surface[54].

From halide electrochemistry[55,56] and the electrical and electrochemical investigations discussed, we can propose a mechanism comprising the following steps: 


\section{WILEY-VCH}

A) $S_{N i O}+I^{-} \rightarrow^{k}{ }_{A}\left[S_{N i O}-I\right]+e^{-}$; the oxidation of iodide at the NiO surface

B) $S_{N i O}^{*}+I^{-} \rightarrow^{k_{B}}\left[S_{N i O}-I\right]+e^{-}$; the oxidation of iodide at the activated NiO surface

C) $S_{N i O} \rightleftharpoons S_{N i O}^{*}$; the potential dependent activation/deactivation of the NiO/PSK interface

D) $\left[S_{N i O}-I\right] \cdot \rightarrow^{k_{D}} I_{P S K}$; the removal of oxidized iodine from the NiO surface

Here $S_{N i O}$ is a pristine surface site of $\mathrm{NiO}$ and $S_{N i O}^{*}$ is an activated one with the rate constant $k_{B}$ higher than $k_{A}, I^{-}$is iodide, $\left[S_{N i O}-I\right]^{\cdot}$ is the iodine radical on the NiO surface after the charge $\left(e^{-}\right)$ transfer and $I_{P S K}$ is the oxidized iodine diffusing into the perovskite. When the potential is increased from the starting value of $-0.1 \mathrm{~V}$, the charge transfer first occurs through step A and, following the potential dependent activation (step C), via step B. The nature of the activated site can only be speculated here. It can be a chemisorbed iodide $\left[S_{N i O}-I\right]^{-}$, as reported for $\mathrm{NiO} / \mathrm{MAPbI}_{3}$ interface[57], or the kinetic barrier reduction could be caused by the proximity of reacted sites $\left[S_{N i O}-I\right]$, i.e. nuclei/clusters of $\mathrm{I}_{2}$. Alternatively, the activation may concern the $\mathrm{NiO}$ alone (e.g. by depopulating the electrons in its trap states[48,56]), however the universality of non-capacitive hysteresis with respect to hole selective materials strongly suggest that iodide is involved in the activation process. As mentioned, iodide might enhance the interface reactivity by also migrating towards the electrode. Step D is the diffusion of oxidized iodine back into the perovskite film. Here we refer to " $I_{P S K}$ " to account for the articulate valence chemistry of iodine and polyiodide (or polyhalide) compounds $\left(\mathrm{I}_{2}^{-}, \mathrm{I}_{3}^{-}\right.$and the mixed halide respective). We recall that $\mathrm{I} \cdot$ diffusion has been documented and is supposed to occur mainly through site exchange between interstitial iodine $\left(I_{i}^{X}\right)$ and lattice iodide $\left(I_{I}^{X}\right)$ with hole exchange[19,58], so involving the lead halide sublattice. 
WILEY-VCH
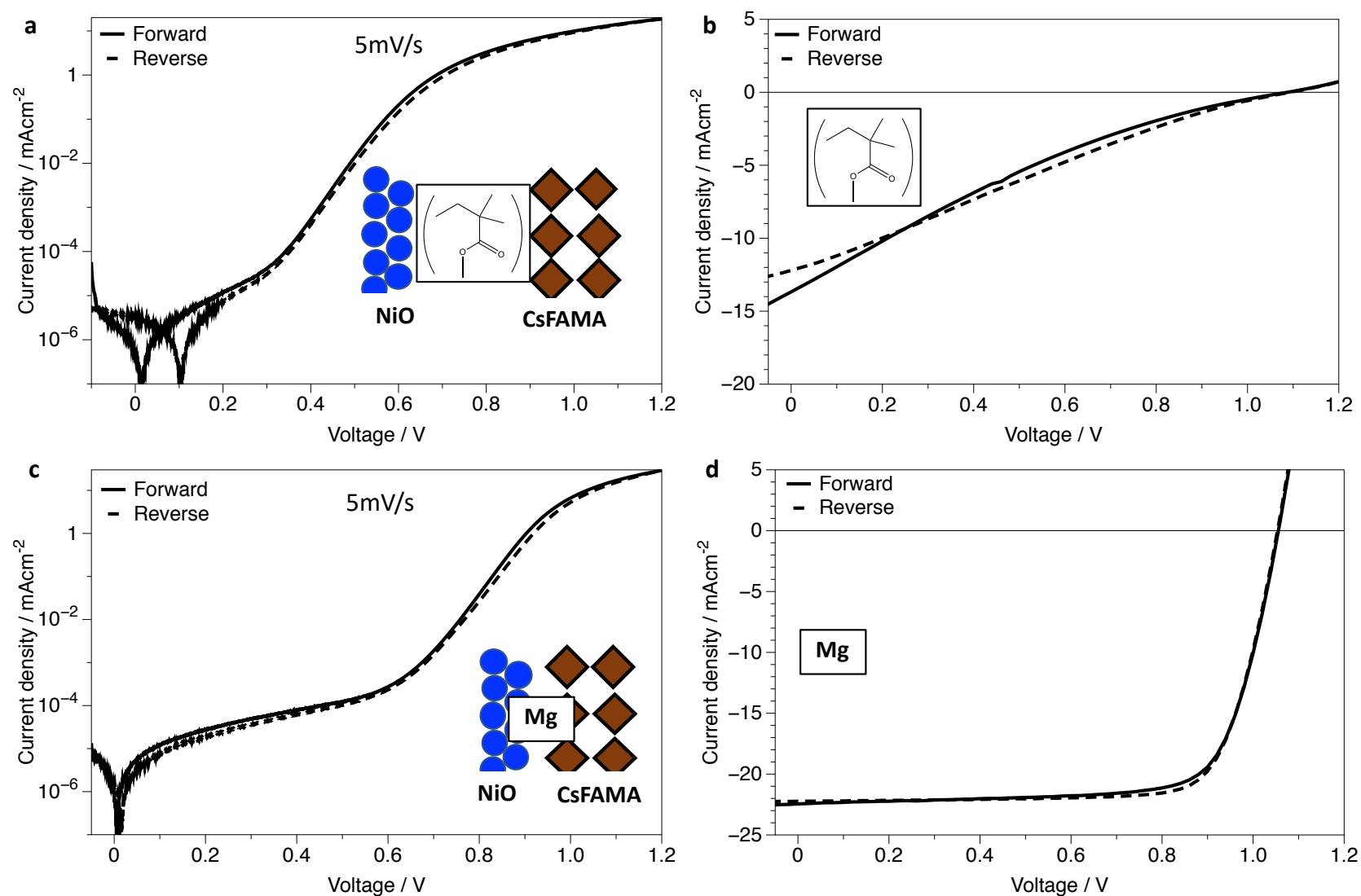

Figure 3 a), b) dark current at $5 \mathrm{mV} / \mathrm{s}$ and JV under AM 1.5G when employing PMMA as an interlayer between perovskite and $\mathrm{NiO}$. c), d) dark current at $5 \mathrm{mV} / \mathrm{s}$ and $\mathrm{JV}$ under $\mathrm{AM} 1.5 \mathrm{G}$ when applying $\mathrm{MgO}(\mathrm{R})$ as the interlayer. Both approaches could eliminate hysteresis, but with PMMA, the device efficiency was sharply reduced, while with $\mathrm{MgO}(\mathrm{R})$ it was slightly increased.

The introduction of a buffer layer can be a promising solution to improve the undesirable interaction between $\mathrm{NiO}$ and perovskite. We explored different materials, relying on the noncapacitive hysteresis as a figure of merit. The first interlayer we discuss is PMMA, cast on NiO via spin-coating from a toluene solution. It has been demonstrated that PMMA can passivate undercoordinated $\mathrm{Pb}^{2+}$ by the interaction of the carbonyl $(\mathrm{C}=\mathrm{O})$ groups, leading to a reduction of the interface recombination[59]. Therefore, PMMA might be considered as the polymeric spacer model to avoid detrimental interaction at the PSC interfaces. With a concentration of $2 \mathrm{mg} / \mathrm{mL}$, it is possible to suppress the non-capacitive hysteresis (Figure 3a) entirely. Unfortunately, the effect of PMMA's low conductivity on series resistance of PSCs is dramatic, with the PCE falling below $4 \%$ and a complete loss of photodiode-like current behaviour (Figure 3b). Reducing the thickness of PMMA to 


\section{WILEY-VCH}

compromise between non-capacitive hysteresis suppression and fast hole transfer under photovoltaics operation was unsuccessful. The PCE loss remained too large, and the dark JV curve assumed a complex shape, with non-capacitive hysteresis overlapping with an evident occurrence of S-shape at high voltages (Figure S6). Moreover, we generally observed poor reproducibility, with significant variations in PV parameters and dark JV features not only across different substrates but also for different pixels on the same substrate. A possible reason for that is the partial solubility of PMMA in DMF and DMSO along with its glass transition temperature $\left(\mathrm{T}_{\mathrm{G}}\right)$ around $100^{\circ} \mathrm{C}$, which is also the annealing temperature of the perovskite film. Nonetheless, these findings, and the correlation between PMMA thickness and non-capacitive hysteresis suppression, further prove that the cause for this hysteresis is the interaction between perovskite and the hole selective materials.

Inorganic insulators represent an interesting alternative to polymers in modifying the interaction between perovskite and the selective contacts[60]. We modified a low-temperature process for $\mathrm{MgO}$ reported previously in literature[61] (Figure S7), and we achieved complete suppression of the noncapacitive hysteresis without reducing device efficiency, as shown in Figures $3 \mathrm{c}$ and $3 \mathrm{~d}$. The interlayer was spin-coated from an ethanol solution of $\mathrm{Mg}(\mathrm{AcO})_{2} 4 \mathrm{H}_{2} \mathrm{O}$, then annealed at $200^{\circ} \mathrm{C}$. At this temperature, not all the organic ligands are removed as discussed in the following. Therefore we will refer to the interlayer as $\mathrm{MgO}(\mathrm{R})$. At high $\mathrm{Mg}(\mathrm{AcO})_{2} 4 \mathrm{H}_{2} \mathrm{O}$ concentrations $(50 \mathrm{mM}$ and $25 \mathrm{mM})$, the interlayers were completely insulating. By reducing the precursor concentration, the photovoltaic behaviour of PSCs was recovered (Figures S7 and S8). In particular, from a $3.5 \mathrm{mM}$ solution we obtained a best device with $\mathrm{Jsc}=22.2 \mathrm{~mA} / \mathrm{cm}^{2}, \mathrm{Voc}=1.055 \mathrm{~V}, \mathrm{FF}=77 \%$ and $\mathrm{PCE}=18.0 \%$, outperforming the reference device $(\mathrm{PCE}=16.8 \%)$. The elimination of non-capacitive hysteresis, together with improved performance, demonstrated the effectiveness of this approach. Therefore, we investigated in more detail the perovskite film, the interlayer chemistry and its effect on device optoelectronics. 
a

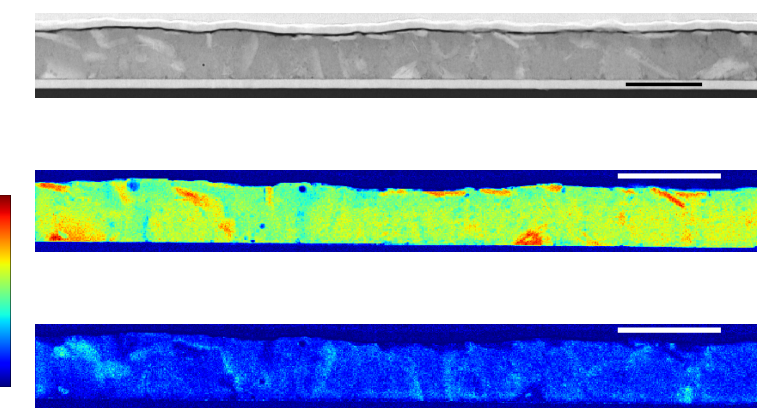

b

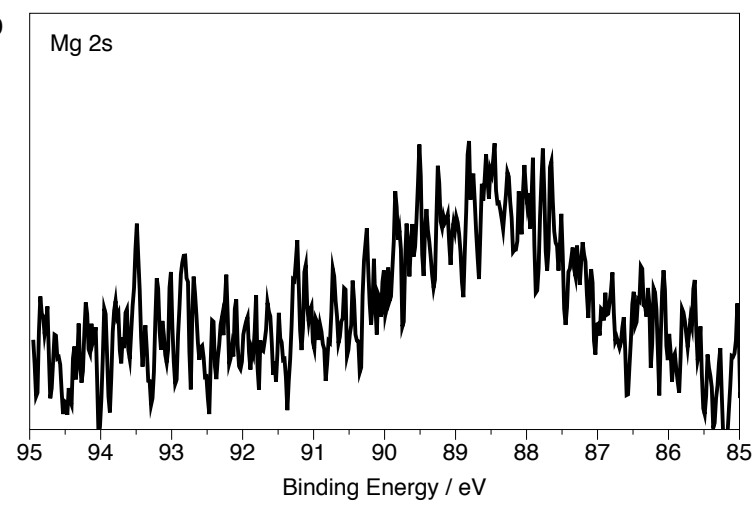

d

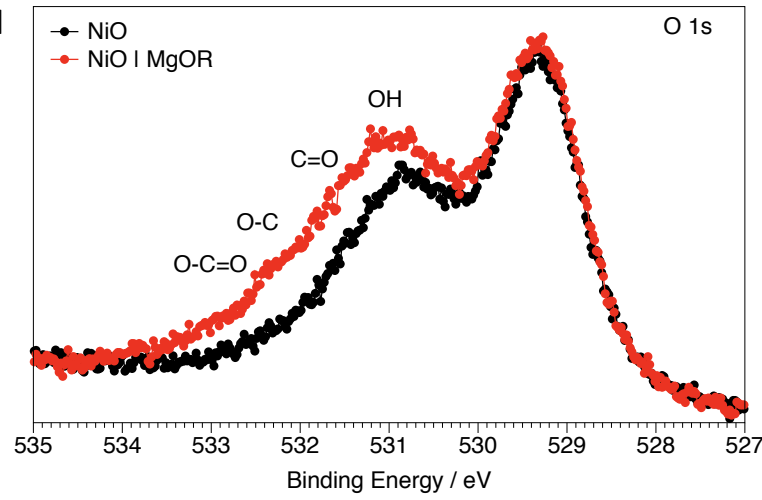

$\mathrm{C} 60 / \mathrm{BCP} / \mathrm{Cu}$

$\mathrm{NiO} / \mathrm{MgOR} / \mathrm{CsFAMA}$

CsFAMA

ITO/HSL

NMF \#1

0-0.1

NMF \#2 0-0.135
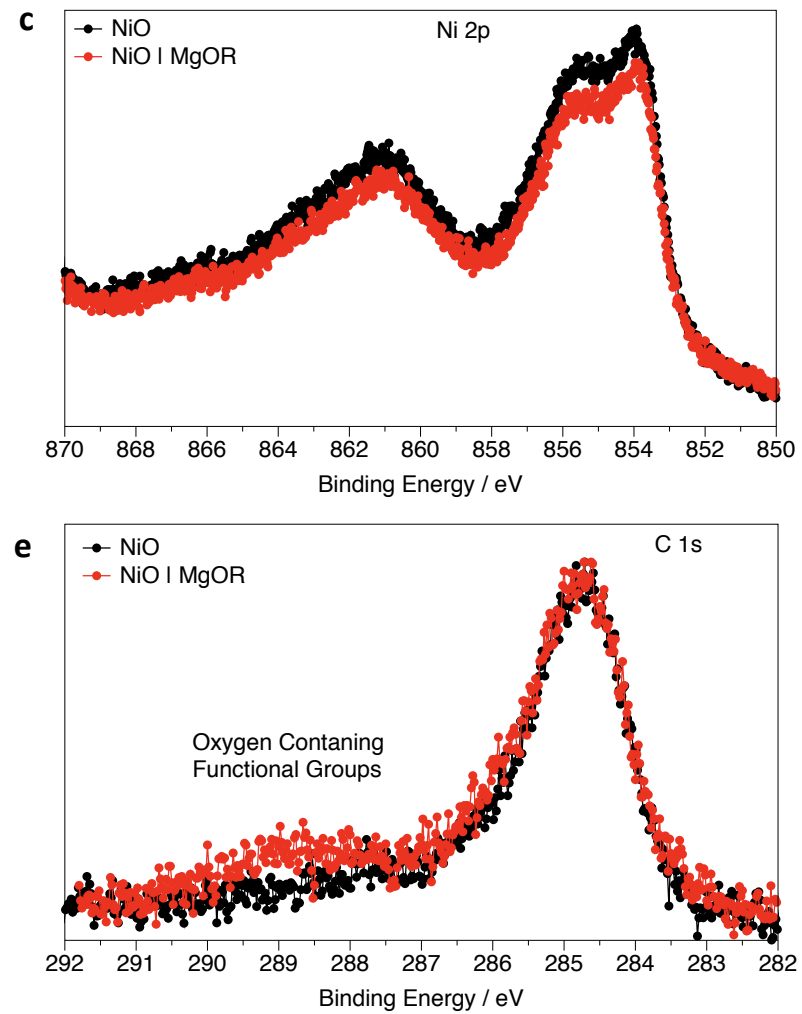

Figure 4. a) Cross-sectional STEM images and STEM-EDX maps of devices without (left) and with (right) an $\mathrm{MgO}(\mathrm{R})$ interlayer. From top to bottom, these are STEM-HAADF images, NMF \#1 (perovskite and $\mathrm{PbI}_{2}$ ) and $\mathrm{NMF}_{\# 2}$ $\left(\mathrm{PbBr}_{2}\right)$ maps. Numbers below the NMF labels refer to minimum and maximum values of the colour range. Layers in the HAADF images are, from top to bottom, $\mathrm{Cu}$ (light grey), $\mathrm{C}_{60}$ (black), perovskite (dark grey), ITO (light grey), and glass (black). The $\mathrm{NiO}$ and $\mathrm{MgO}(\mathrm{R})$ layers are too thin to be visible. Scale bars are $0.5 \mu \mathrm{m} . \mathbf{b}), \mathbf{c})$, d) and e) XPS spectra of Mg 1s for the $\mathrm{MgO}(\mathrm{R})$-coated $\mathrm{NiO}$, and $\mathrm{Ni} 2 \mathrm{p}, \mathrm{O}$ 1s and $\mathrm{C}$ 1s for $\mathrm{NiO}$ and the $\mathrm{MgO}(\mathrm{R})$-coated $\mathrm{NiO}$ samples.

By introducing an interlayer on $\mathrm{NiO}$, we are modifying the substrate for perovskite growth, which might affect its quality. From scanning electron microscopy (SEM) top view and cross-sectional micrographs (Figure S9), the morphology appears to be unaffected by the introduction of an $\mathrm{Mg}$ based interlayer. The two films are both composed of large grains with a diameter above $400 \mathrm{~nm}$. We 


\section{WILEY-VCH}

also conducted a scanning transmission electron microscopy (STEM) analysis on cross-sections of full devices to characterise the compositional distribution within the two perovskite films. Knowing this compositional distribution is essential as non-uniformity on the nano-/micro- scale affects the device optoelectronics, as highlighted in recent works[62,63]. The top row of Figure 4a shows crosssectional high-angle annular dark field (HAADF) images for the two samples. From these images, we can state that the two films appear similar, both having bright grains interspersed within the perovskite film. Since the contrast of HAADF images is approximately proportional to the square of effective atomic number $\left(Z_{E f f}{ }^{2}\right)$, we know that these bright grains must have a higher share of heavy elements than the bulk perovskite. We then collected energy dispersive X-ray spectroscopy (EDX) spectrum images (SIs) to investigate the chemical composition of the perovskite layer. EDX SIs were acquired with low beam current and dwell time to minimise beam damage. These SIs were then treated with two multivariate statistical analysis algorithms, namely principal component analysis for denoising and non-negative matrix factorisation (NMF) for decomposition into easily interpretable components $^{[64]}$. From NMF decomposition, we identified two distinct compositions for the bright grains in addition to the bulk perovskite layer, hereafter referred to as NMF \#1 and NMF \#2. Most of these grains appear together with the bulk perovskite in one component, NMF \#1, which is composed of lead and iodine as shown in this component's spectrum (figure S13 a). Therefore, the most likely identity of these grains is $\mathrm{PbI}_{2}$. The other grains form a separate NMF component, NMF \#2, whose spectrum contains lead and bromine peaks (figure $\mathrm{S} 13$ b) pointing to $\mathrm{PbBr}_{2}$ as a possible stoichiometry. As reported by Correa-Baena et al., halide non-uniformity is a common feature of multi-cation multi-anion perovskite compositions[62]. For our discussion, the critical result is that the non-capacitive hysteresis appears to be uncorrelated to compositional homogeneity, i.e. the quality, of the bulk perovskite.

We conducted an X-ray photoelectron spectroscopy (XPS) investigation to shed light on the chemical composition of the Mg-based interlayer. The weak Mg 1s core level signal reported in Figure $4 \mathrm{~b}$ points to a thickness of a few atomic layers, as confirmed by the slight attenuation of the 


\section{WILEY-VCH}

Ni $2 p$ core level, Figure $4 c$. The latter signal is composed of different peaks due to the various mechanisms of relaxation upon Ni photoionisation, which are due to the active electronic correlation affecting its band structure[65,66]. We cannot entirely exclude the possibility of surface doping even if the low-temperature treatment and very similar shape of the $\mathrm{Ni} 2 \mathrm{p}$ core level point to the low likelihood of this hypothesis. From O 1s (Figure 4d) and C 1s (Figure 4e) core levels, we can infer that a considerable amount of organic ligands remain in the film. The $\mathrm{O} 1 \mathrm{~s}$ spectrum for $\mathrm{NiO}$ is composed of the peak from the oxygen in the NiO lattice $(529.8 \mathrm{eV})$, a peak for surface $\mathrm{OH}(531.2$ $\mathrm{eV}$ ), and a tail at higher binding energies which is generally attributed to weakly bonded oxygen ${ }^{[65]}$. After introducing the interlayer, the XPS signal markedly increased in the binding energy range

typical of $\mathrm{C}=\mathrm{O}, \mathrm{C}-\mathrm{O}$ and $\mathrm{O}=\mathrm{C}-\mathrm{O}$ functional groups (Figure $\mathrm{S} 10$ ). The $\mathrm{C} 1 \mathrm{~s}$ spectrum of the $\mathrm{Mg}$-coated $\mathrm{NiO}$ shows a broad feature at higher binding energies compatible with the mentioned functional groups, while only the adventitious carbon is found on NiO. Overall, the XPS investigation revealed that the organic ligands, i.e. ethoxy and acetate anions from the solution, remained on the surface after thermal annealing at $200^{\circ} \mathrm{C}$. 

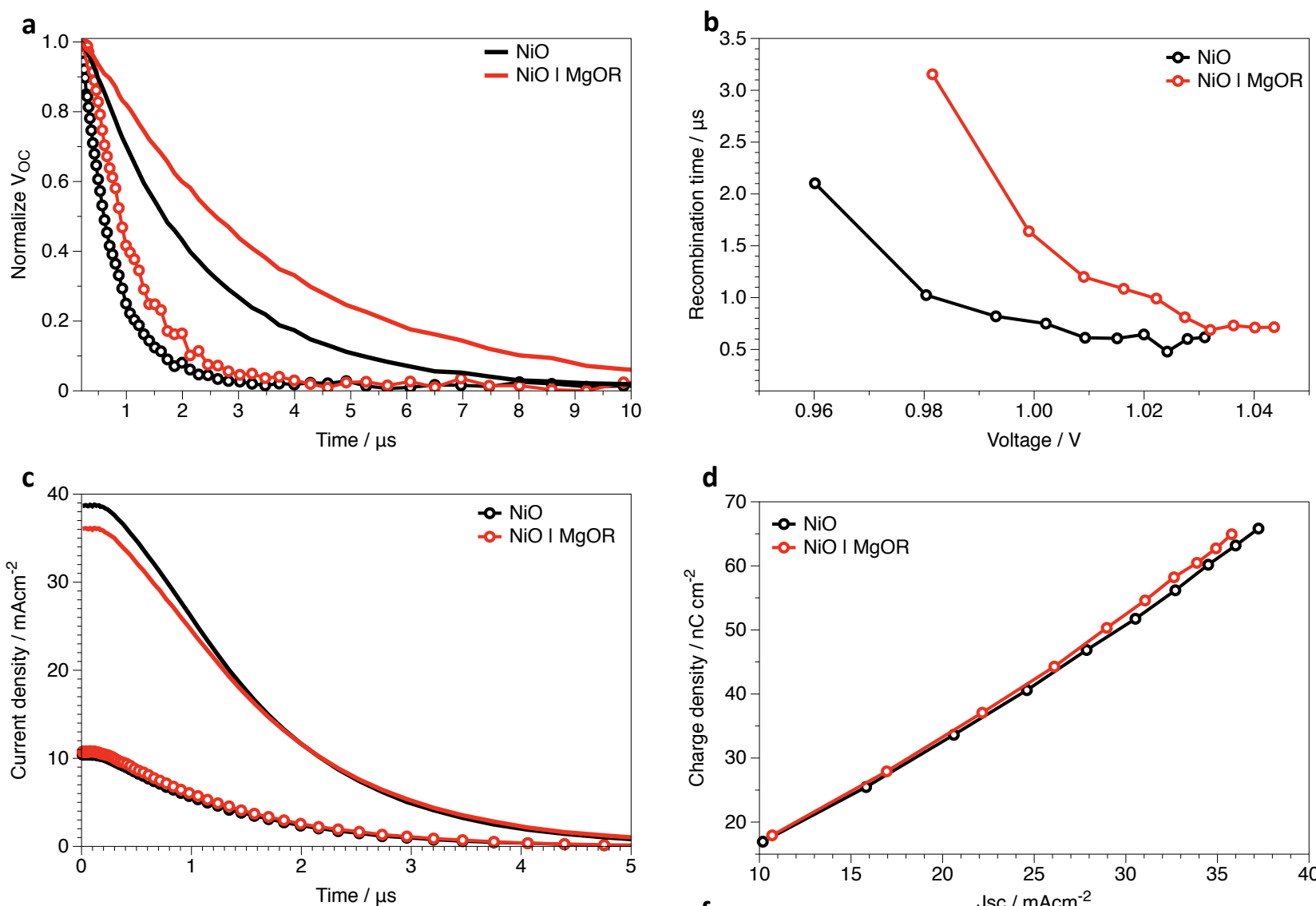

d
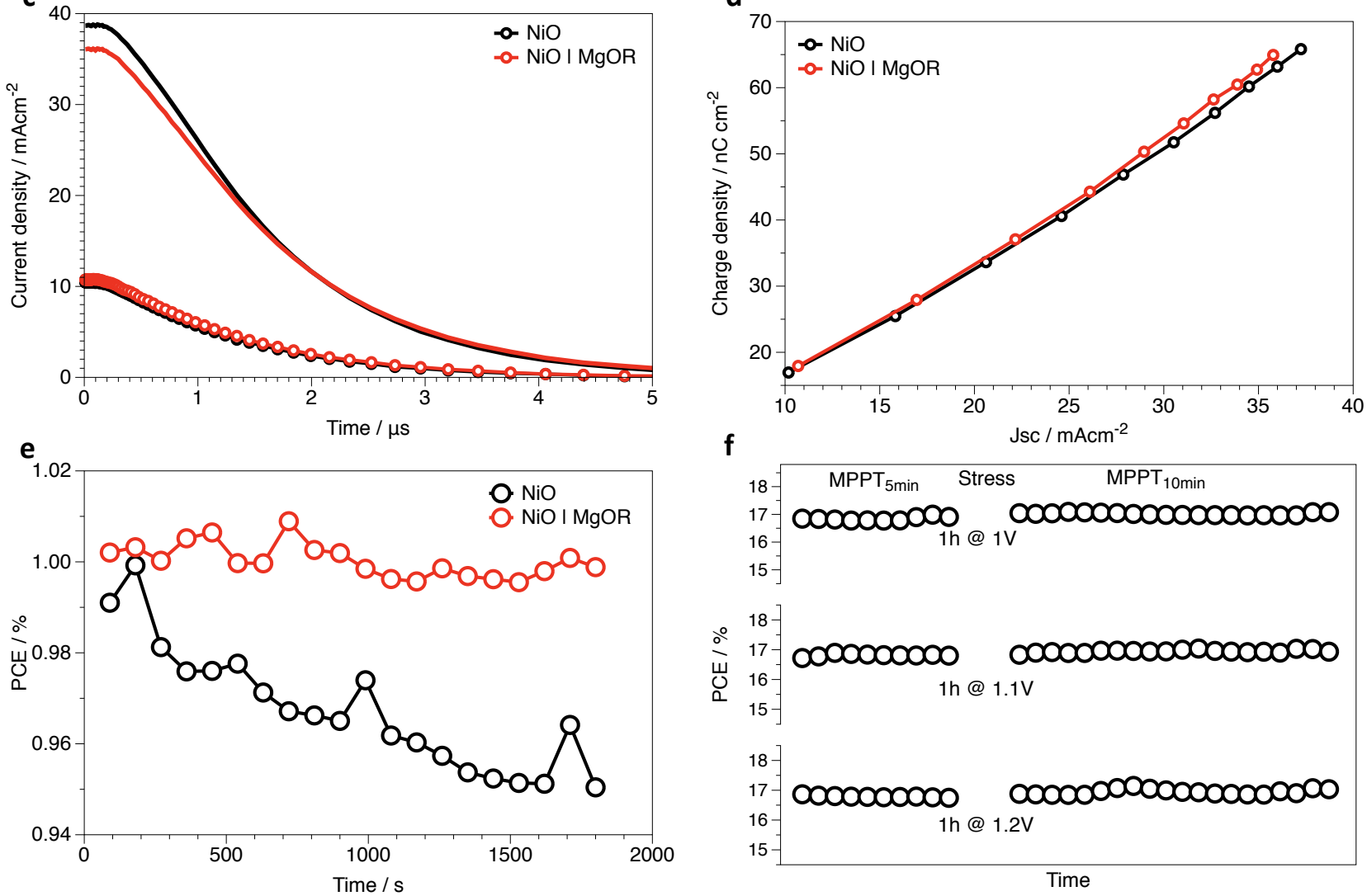

f
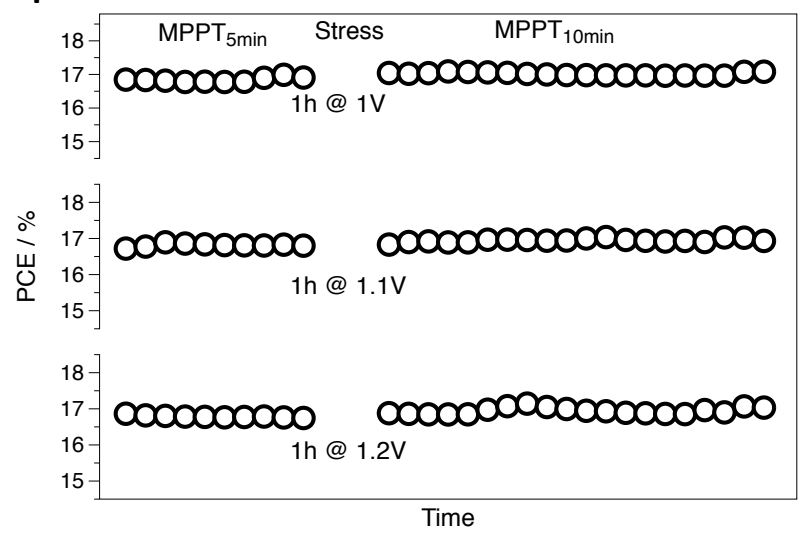

Figure 5. a) and b) Voc decay and the derived recombination lifetime at different light intensities (continuous line: 0.1 Sun equivalent intensity; circles: 1.8 Sun equivalent intensity) from TPV experiments on PSCs employing NiO and $\mathrm{NiO} \mid \mathrm{MgO}(\mathrm{R})$ as HSLs. c) and d) Jsc decay and the derived charge accumulation at different light intensities (continuous line: 0.5 Sun equivalent; circles: 1.8 Sun equivalent) from CE experiments on the same PSCs as in a) and b). e) 30 minutes of MPPT in $\mathrm{N}_{2}$ atmosphere under AM 1.5G for the PSCs employing $\mathrm{NiO}$ and $\mathrm{NiO} \mid \mathrm{MgO}(\mathrm{R})$ as HSL. f) Stability of the MPPT of $\mathrm{NiO} \mid \mathrm{MgO}(\mathrm{R})$ based PSCs after alternating MPPT and electrical bias in the dark (from 1.0 to $1.2 \mathrm{~V}$ for $1 \mathrm{~h}$ ). We tracked the maximum power point for 5 minutes before the stress and for 10 minutes after that. 


\section{WILEY-VCH}

Using transient photovoltage (TPV) and charge extraction (CE) employing a white LED (5000 $\mathrm{K})$, we investigated how the $\mathrm{MgO}(\mathrm{R})$ interlayer affects charge recombination and transfer at the $\mathrm{NiO}$ | CsFAMA interface. In TPV the device is kept at open circuit. A $2 \mu$ s light pulse was applied over a background illumination and the rise and subsequent fall of the Voc was tracked. The measurement is repeated at different light intensities. In the whole protocol, no charge is extracted from the device, therefore the decaying transient of the Voc is attributable to the internal recombination in the bulk of perovskite and at the interfaces with selective contacts[67]. As we report in Figures 5a,b, the introduction of $\mathrm{MgO}(\mathrm{R})$ led to a substantial increase of recombination lifetime, pointing to an improved quality of the $\mathrm{NiO}$ interface. In $\mathrm{CE}$ the device is kept at short circuit and, upon applying again a $2 \mu \mathrm{s}$ light pulse (from the dark condition in this case), the photocurrent is recorded. When the pulse ended, no additional photocarriers is generated, therefore the current decayed as carriers were extracted from the device (figure 5c). Depending on the illumination intensity, the transient Jsc from $\mathrm{MgO}(\mathrm{R})$-based devices can be higher (low illumination) or smaller (high illumination) than for $\mathrm{NiO}$ (Figure 5d). Moreover, at high illumination, a larger accumulation of charge is observed for $\mathrm{MgO}(\mathrm{R})$, as outlined from the higher slope for the $\mathrm{NiO} \mid \mathrm{MgO}(\mathrm{R})$ device in Figure 5d. Combining the two results, we can state that the insulating nature of the $\mathrm{MgO}(\mathrm{R})$ interlayer hinders the hole extraction and results in larger charge accumulation in the device, under strong illumination. However, the reduction of interface recombination largely prevails at lower light intensities (and notably around 1 Sun), yielding higher current despite the insulating nature of the interlayer. Furthermore, the power output stability improved with $\mathrm{MgO}(\mathrm{R})$. Notably, already in the firsts 30 minutes of tracking the improvement upon the introduction of $\mathrm{MgO}(\mathrm{R})$ can be appreciated. As shown in Figure 5e, the power output is stable with the $\mathrm{MgO}(\mathrm{R})$ interlayer while for $\mathrm{NiO}$ we observed some instability in the PCE and a (small) decreasing slope from the beginning of the tracking. More interestingly, the stable output with $\mathrm{MgO}(\mathrm{R})$ layer was preserved when alternating MPP tracking under light with prolonged $(1 \mathrm{~h})$ bias in dark at voltages between 1.0 and $1.2 \mathrm{~V}$ (figure $5 \mathrm{f}$ ). This test was inspired by recent papers on the resistance of PSCs to electrical bias. For instance, Jung[32] observed a strong increase of hysteresis and loss of PCE upon biasing at $0.95 \mathrm{~V}$ for 1 hour, and a similar effect was reported when biasing 


\section{WILEY-VCH}

$\mathrm{NiO}\left|\mathrm{CH}_{3} \mathrm{NH}_{3} \mathrm{PbI}_{3}\right| \mathrm{PCBM}$ PSCs at $1 \mathrm{~V}[68]$. These results are somewhat similar to the loss we observed

for $\mathrm{NiO}$ devices, and that were suppressed with the $\mathrm{MgO}(\mathrm{R})$ interlayer, as we show in figure $\mathrm{S} 12$. 


\section{WILEY-VCH}

\section{Conclusions}

The non-capacitive hysteresis in dark in NiO-based p-i-n PSCs has been investigated. This is due to the electrical bias induced instability at the $\mathrm{NiO} /$ Perovskite interface, linked to the electrochemical reactivity of the perovskite layer. With a moderate forward bias (below the maximum power point) the degradation is already triggered and the hysteresis is detectable, with the migration of electroactive species toward the HSL and an enhancement of the reactivity of the NiO / CsFAMA interface accounting for that. This is detrimental to device stability. Two different interface engineering approaches to address this problem are reported. Employing PMMA, we needed to introduce a thick buffer layer to suppress the non-capacitive hysteresis, bringing into the device a robust unwanted enhancement of the series resistance. This behaviour resulted in a severe loss in PCE, which dropped from 17\% to below 4\%. Employing a hybrid Mg-organic $(\mathrm{MgO}(\mathrm{R}))$ interlayer, we were able to suppress the hysteresis without sacrificing the PCE. We showed that the $\operatorname{MgO}(\mathrm{R})$ layer reduces the interface recombination and enabled PCEs of up to $18 \%$. More importantly, the MPP tracking revealed that suppressing the hysteresis also stabilised the power output, even after electrical stress in the dark. Future investigations that could be inspired by this work are:

- Understanding the microscopic mechanism of the redox process between perovskite and selective contacts, including the interplay between interface reactivity and ionic migration, and the identification of the relaxation process.

- $\quad$ Finding new buffer layers working in both p-i-n and n-i-p architecture.

Finally, we recommend analysing the hysteresis in dark conditions at different scan rates to have insights into the interface stability against electrical bias, especially when introducing new hole selective materials, which is a very topical issue in perovskite photovoltaics. The lack of noncapacitive hysteresis might be a compelling figure of merit for the electrical stability in the forward bias of the p-type interface. 


\section{WILEY-VCH}

\section{Acknowledgments}

D.D.G. acknowledges the PhD program in Chemistry from the Department of Chemistry at the University of Rome La Sapienza. A.A. acknowledges financial support from National Natural Science Foundation of China; grant number 21750110442. A.D.C. acknowledges the financial support from Ministry of Education and Science of the Russian Federation in the framework of Megagrant No. 14.Y26.31.0027. The work of G.C. and L.K. was supported by the German Federal Ministry for Economic Affairs and Energy within the research cluster "Persist" under Grant 0324037C. The authors acknowledges the EMIL (Energy Materials In-situ Laboratory Berlin) Lab and R.Wilks and J.Frisch for the technical support for the XPS investigation.

\section{Supporting Information}

Supporting Information is available from the Wiley Online Library. The experimental section together with additional electrical and electrochemical investigation are provided. Further, characterization of the perovskite morphology and details on the device and interlayer optimization can be found.

\section{References}

[1] “NREL Best Research-Cell Efficiencies," can be found under https://www.nrel.gov/pv/cellefficiencies.html

[2] M. Saliba, M. Stolterfoht, C. M. Wolff, D. Neher, A. Abate, Joule 2018, 2, 1019.

[3] Q. Wang, N. Phung, D. Di Girolamo, P. Vivo, A. Abate, 2018, 12, 865-886.

[4] A. M. A. Leguy, Y. Hu, M. Campoy-Quiles, M. I. Alonso, O. J. Weber, P. Azarhoosh, M. Van Schilfgaarde, M. T. Weller, T. Bein, J. Nelson, P. Docampo, P. R. F. Barnes, Chem. Mater. 2015, 27, 3397.

[5] Z. Song, A. Abate, S. C. Watthage, G. K. Liyanage, A. B. Phillips, U. Steiner, M. Graetzel, M. J. Heben, Adv. Energy Mater. 2016, 6, DOI 10.1002/aenm.201600846.

[6] D. Di Girolamo, M. I. Dar, D. Dini, L. Gontrani, R. Caminiti, A. Mattoni, M. Grätzel, S. Meloni, J. Mater. Chem. A 2019,7, 12292-12302.

[7] N. Aristidou, C. Eames, I. Sanchez-Molina, X. Bu, J. Kosco, M. Saiful Islam, S. A. Haque, Nat. Commun. 2017, 8, 1.

[8] N. Aristidou, I. Sanchez-Molina, T. Chotchuangchutchaval, M. Brown, L. Martinez, T. Rath, S. A. Haque, Angew. Chemie - Int. Ed. 2015, 54, 8208. 
[9] D. Bryant, N. Aristidou, S. Pont, I. Sanchez-Molina, T. Chotchunangatchaval, S. Wheeler, J. R. Durrant, S. A. Haque, Energy Environ. Sci. 2016, 9, 1655.

[10] F. Matteocci, L. Cinà, E. Lamanna, S. Cacovich, G. Divitini, P. A. Midgley, C. Ducati, A. Di Carlo, Nano Energy 2016, 30, 162.

[11] R. T. Ginting, M. K. Jeon, K. J. Lee, W. Y. Jin, T. W. Kim, J. W. Kang, J. Mater. Chem. A $2017,5,4527$.

[12] B. Brunetti, C. Cavallo, A. Ciccioli, G. Gigli, A. Latini, Sci. Rep. 2016, 6, 1.

[13] F. Matteocci, Y. Busby, J.-J. Pireaux, G. Divitini, S. Cacovich, C. Ducati, A. Di Carlo, ACS Appl. Mater. Interfaces 2015, 7, 26176.

[14] K. Domanski, J. P. Correa-Baena, N. Mine, M. K. Nazeeruddin, A. Abate, M. Saliba, W. Tress, A. Hagfeldt, M. Grätzel, ACS Nano 2016, 10, 6306.

[15] G. Divitini, F. Matteocci, A. Di Carlo, P. A. Midgley, S. Cacovich, C. Ducati, L. Ciná, Nanoscale 2017, 9, 4700 .

[16] E. T. Hoke, D. J. Slotcavage, E. R. Dohner, A. R. Bowring, H. I. Karunadasa, M. D. McGehee, Chem. Sci. 2015, 6, 613.

[17] S. Cacovich, G. Divitini, F. Matteocci, Y. Busby, J.-J. Pireaux, A. Di Carlo, C. Ducati, Eur. Microsc. Congr. 2016 Proc. 2016, 812.

[18] B. Roose, C. M. Johansen, K. Dupraz, T. Jaouen, P. Aebi, U. Steiner, A. Abate, J. Mater. Chem. A 2018, 6, 1850-1857.

[19] G. Y. Kim, A. Senocrate, T. Y. Yang, G. Gregori, M. Grätzel, J. Maier, Nat. Mater. 2018, 17, 445.

[20] D. Meggiolaro, S. G. Motti, E. Mosconi, A. J. Barker, J. Ball, C. Andrea Riccardo Perini, F. Deschler, A. Petrozza, F. De Angelis, Energy Environ. Sci. 2018, 11, 702.

[21] C. Li, A. Guerrero, Y. Zhong, A. Gräser, C. A. M. Luna, J. Köhler, J. Bisquert, R. Hildner, S. Huettner, Small 2017, 13, 1.

[22] S. Y. Luchkin, A. F. Akbulatov, L. A. Frolova, M. P. Griffin, A. Dolocan, R. Gearba, D. A. V. Bout, P. A. Troshin, K. J. Stevenson, ACS Appl. Mater. Interfaces 2017, 9, 33478. 


\section{WILEY-VCH}

[23] V. Kumar, J. Barbé, W. L. Schmidt, K. Tsevas, B. Ozkan, C. M. Handley, C. L. Freeman, D. C. Sinclair, I. M. Reaney, W. C. Tsoi, A. Dunbar, C. Rodenburg, J. Mater. Chem. A 2018, 6, 23578 .

[24] E. L. Unger, E. T. Hoke, C. D. Bailie, W. H. Nguyen, A. R. Bowring, T. Heumüller, M. G. Christoforo and M. D. McGehee, Energy Environ. Sci. 2014, 7, 3690.

[25] H. J. Snaith, A. Abate, J. M. Ball, G. E. Eperon, T. Leijtens, N. K. Noel, S. D. Stranks, J. T. W. Wang, K. Wojciechowski, W. Zhang, J. Phys. Chem. Lett. 2014, 5, 1511.

[26] K. Domanski, B. Roose, T. Matsui, M. Saliba, S. H. Turren-Cruz, J. P. Correa-Baena, C. R. Carmona, G. Richardson, J. M. Foster, F. De Angelis, J. M. Ball, A. Petrozza, N. Mine, M. K. Nazeeruddin, W. Tress, M. Grätzel, U. Steiner, A. Hagfeldt, A. Abate, Energy Environ. Sci. 2017, 10, 604 .

[27] Q. Jeangros, M. Duchamp, J. Werner, M. Kruth, R. E. Dunin-Borkowski, B. Niesen, C. Ballif, A. Hessler-Wyser, Nano Lett. 2016, 16, 7013.

[28] G. Kakavelakis, I. Paradisanos, B. Paci, A. Generosi, M. Papachatzakis, T. Maksudov, L. Najafi, A. Esaú, D. Rio, G. Kioseoglou, E. Stratakis, F. Bonaccorso, E. Kymakis, 2018, $1702287,1$.

[29] V. Roiati, E. Mosconi, A. Listorti, S. Colella, G. Gigli, F. De Angelis, Nano Lett. 2014, 14, 2168.

[30] J. Carrillo, A. Guerrero, S. Rahimnejad, O. Almora, I. Zarazua, E. Mas-Marza, J. Bisquert, G. Garcia-Belmonte, Adv. Energy Mater. 2016, 6, 1.

[31] R. A. Kerner, B. P. Rand, J. Phys. Chem. Lett. 2017, 8, 2298.

[32] H. J. Jung, D. Kim, S. Kim, J. Park, V. P. Dravid, B. Shin, Adv. Mater. 2018, 1802769, 1802769.

[33] B. Roose, R. H. Friend, Adv. Mater. Interfaces 2019, 1801788, 1.

[34] O. Almora, C. Aranda, I. Zarazua, A. Guerrero, G. Garcia-Belmonte, ACS Energy Lett. 2016, $1,209$.

[35] M. B. Islam, M. Yanagida, Y. Shirai, Y. Nabetani, K. Miyano, ACS Omega 2017, 2, 2291. 


\section{WILEY-VCH}

[36] J. W. Jo, M. S. Seo, J. W. Jung, J. S. Park, B. H. Sohn, M. J. Ko, H. J. Son, J. Power Sources 2018, 378, 98 .

[37] D. Saranin, P. Gostischev, D. Tatarinov, I. Ermanova, V. Mazov, Dmitry Muratov, A. Tameev, D. Kuznetsov, S. Didenko, A. Di Carlo, Materials (Basel). 2019, $12(9), 1406$.

[38] Q. Wang, C. C. Chueh, T. Zhao, J. Cheng, M. Eslamian, W. C. H. Choy, A. K. Y. Jen, ChemSusChem 2017, 10, 3794.

[39] J. Zhang, H. Luo, W. Xie, X. Lin, X. Hou, J. Zhou, S. Huang, W. Ou-Yang, Z. Sun, X. Chen, Nanoscale 2018, 10, 5617.

[40] O. Almora, I. Zarazua, E. Mas-Marza, I. Mora-Sero, J. Bisquert, G. Garcia-Belmonte, J. Phys. Chem. Lett. 2015, 6, 1645.

[41] G. Garcia-belmonte, U. Jaume, J. Bisquert, U. Jaume, ACS Energy Lett. 2016, DOI 10.1021/acsenergylett.6b00293.

[42] M. Valles-Pelarda, B. C. Hames, I. García-Benito, O. Almora, A. Molina-Ontoria, R. S. Sánchez, G. Garcia-Belmonte, N. Martín, I. Mora-Sero, J. Phys. Chem. Lett. 2016, 7, 4622.

[43] M. Saliba, T. Matsui, J. Y. Seo, K. Domanski, J. P. Correa-Baena, M. K. Nazeeruddin, S. M. Zakeeruddin, W. Tress, A. Abate, A. Hagfeldt, M. Grätzel, Energy Environ. Sci. 2016, 9, 1989.

[44] S. A. L. Weber, I. M. Hermes, S.-H. Turren-Cruz, C. Gort, V. W. Bergmann, L. Gilson, A. Hagfeldt, M. Graetzel, W. Tress, R. Berger, Energy Environ. Sci. 2018, 11, 2404-2413.

[45] A. J. Bard, A. B. Bocarsly, F. R. F. Fan, E. G. Walton, M. S. Wrighton, J. Am. Chem. Soc. 1980, 102,3671 .

[46] D. Moia, I. Gelmetti, P. Calado, W. Fisher, M. Stringer, O. S. Game, Y. Hu, P. Docampo, D. G. Lidzey, E. J. Palomares, J. Nelson, P. R. F. Barnes, Energy Environ. Sci. 2019, DOI 12961308.

[47] A. I. Popov, D. H. Geske, J. Am. Chem. Soc. 1958, 80, 1340.

[48] L. D’Amario, G. Boschloo, A. Hagfeldt, L. Hammarström, J. Phys. Chem. C 2014, 118, 19556. 


\section{WILEY-VCH}

[49] R. Memming, Semiconductor Electrochemistry, Wiley-VHC, 2000.

[50] J. Y. Lee, J. Electrochem. Soc. 2006, 137, 1402.

[51] H. Yang, K. Kwon, T. M. Devine, J. W. Evans, J. Electrochem. Soc. 2002, 147, 4399.

[52] O. Almora, C. Aranda, E. Mas-Marzá, G. Garcia-Belmonte, Appl. Phys. Lett. 2016, 109, DOI $10.1063 / 1.4966127$

[53] I. Mora-Seró, Joule 2018, 2, 585.

[54] M. Bonomo, A. Di Carlo, D. Dini, J. Electrochem. Soc. 2018, 165, H889.

[55] R. P. Akkermans, Q. Fulian, S. L. Roberts, M. F. Suarez, R. G. Compton, J. Phys. Chem. B 1999, 103, 8319 .

[56] M. Bonomo, D. Dini, A. G. Marrani, Langmuir 2016, 32, 11540.

[57] W. Nie, H. Tsai, J. C. Blancon, F. Liu, C. C. Stoumpos, B. Traore, M. Kepenekian, O. Durand, C. Katan, S. Tretiak, J. Crochet, P. M. Ajayan, M. Kanatzidis, J. Even, A. D. Mohite, Adv. Mater. 2018, 30, 1.

[58] L. Zhang, P. H.-L. Sit, J. Mater. Chem. A 2017, 5(45), 23976.

[59] J. Peng, J. I. Khan, W. Liu, E. Ugur, T. Duong, Y. Wu, H. Shen, K. Wang, H. Dang, E. Aydin, X. Yang, Y. Wan, K. J. Weber, K. R. Catchpole, F. Laquai, S. De Wolf, T. P. White, Adv. Energy Mater. 2018, $1801208,1801208$.

[60] M. Kot, L. Kegelmann, P. Kus, N. Tsud, I. Matolinova, S. Albrecht, V. Matolin, D. Schmeisser, C. Das, ChemSusChem 2018, 11, 3640.

[61] J. Dagar, S. Castro-Hermosa, G. Lucarelli, F. Cacialli, T. M. Brown, Nano Energy 2018, 49, 290.

[62] J.-P. Correa-Baena, Y. S. Meng, J. Snaider, N. T. P. Hartono, M. G. Bawendi, M. V. Holt, X. Li, Z. Cai, J. R. Poindexter, M. A. Jensen, T. M. Brenner, Y. Luo, S. Wieghold, L. Nienhaus, S. Wang, T. Wang, B. Lai, T. Buonassisi, S. Sun, L. Huang, D. P. Fenning, Science (80-. ). 2019, 363, 627.

[63] M. Abdi-Jalebi, B. Philippe, S. Cacovich, M. Alsari, C. Stavrakas, E. P. Booker, C. Ducati, E. M. Hutter, H. Rensmo, T. J. Savenije, G. Divitini, S. Lilliu, J. M. Richter, Z. Andaji- 


\section{WILEY-VCH}

Garmaroudi, S. D. Stranks, A. J. Pearson, R. H. Friend, Nature 2018, 555, 497.

[64] S. Cacovich, F. Matteocci, M. Abdi-Jalebi, S. D. Stranks, A. Di Carlo, C. Ducati, G. Divitini, ACS Appl. Energy Mater. 2018, 1, 7174.

[65] A. G. Marrani, V. Novelli, S. Sheehan, D. P. Dowling, D. Dini, ACS Appl. Mater. Interfaces 2014, 6, 143.

[66] P. A. Shen, Z. X., List, R. S., Dessau, D. S., Wells, B. O., Jepsen, O., Arko, A. J., ... \& Lindberg, Phys. Rev. B 1991, 44(8), 3604.

[67] P. Lopez-Varo, J. A. Jiménez-Tejada, M. García-Rosell, S. Ravishankar, G. GarciaBelmonte, J. Bisquert, O. Almora, Adv. Energy Mater. 2018, 8, 1.

[68] J. Barbé, V. Kumar, M. J. Newman, H. K. H. Lee, S. M. Jain, H. Chen, C. Charbonneau, C. Rodenburg, W. C. Tsoi, Sustain. Energy Fuels 2018, 2, 905. 


\section{WILEY-VCH}

TOC

The hysteresis in dark, attributable to bias induced degradation of the p-type interface, is investigated and eliminated in NiO-based inverted perovskite solar cells. Enhanced stability to forward bias is obtained with the introduction of a low-temperature hydridid magnesium-based interlayer.

Keywords Perovskite Solar Cells, Hysteresis, Stability, Interface Engineering, NiO.

Diego Di Girolamo, Fabio Matteocci, Felix Utama Kosasih, Ganna Chistiakova, Weiwei Zuo, Giorgio Divitini, Lars Korte, Caterina Ducati, Aldo Di Carlo, Danilo Dini, Antonio Abate*

Stability and Dark Hysteresis Correlate in NiO-based Perovskite Solar Cells

\section{Graphical TOC}

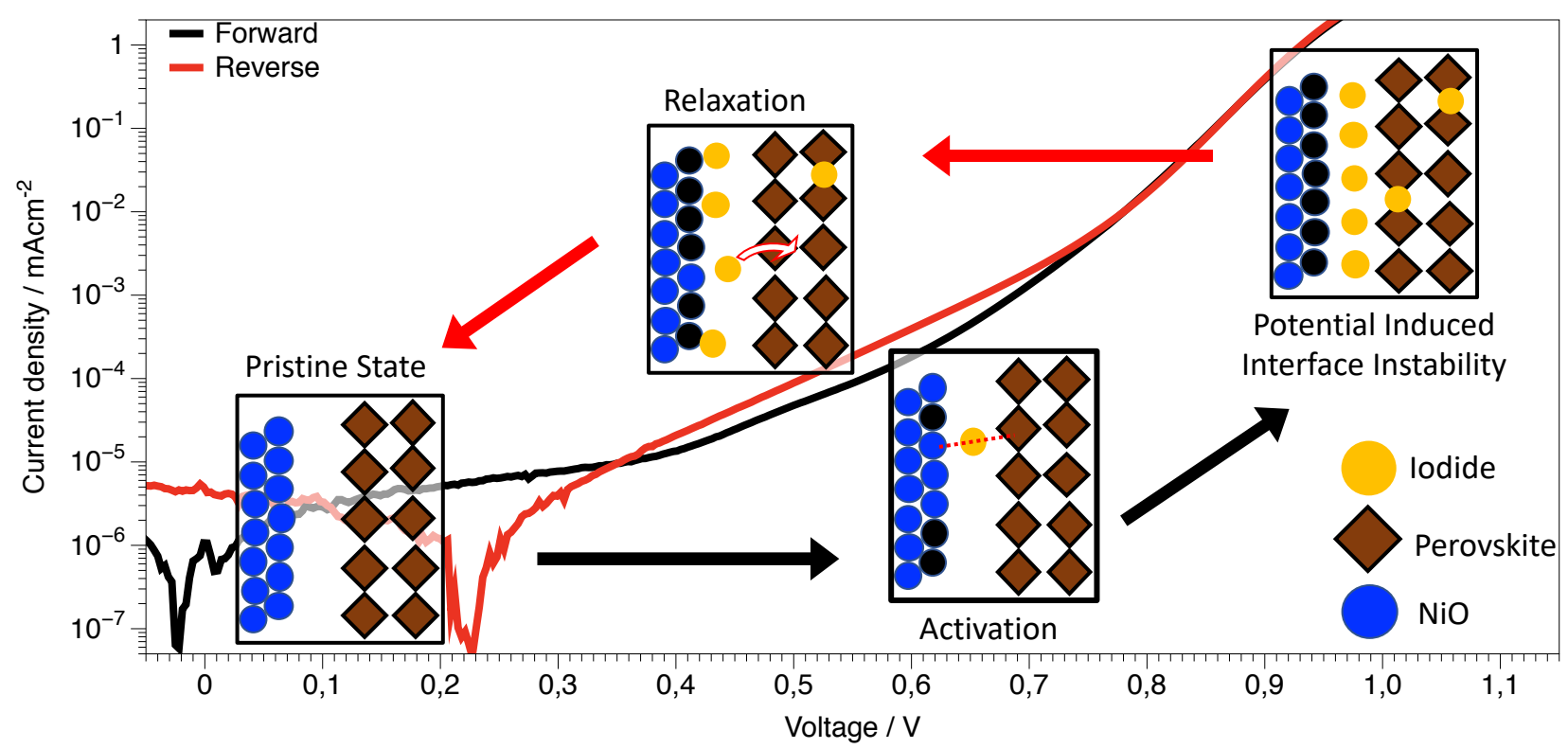




\section{WILEY-VCH}

\section{Supporting Information}

\section{Stability and Dark Hysteresis Correlate in NiO-based Perovskite Solar Cells}

Diego Di Girolamo, Fabio Matteocci, Felix Utama Kosasih, Ganna Chistiakova, Weiwei Zuo, Giorgio Divitini, Lars Korte, Caterina Ducati, Aldo Di Carlo, Danilo Dini, Antonio Abate*

Diego Di Girolamo, Prof. Danilo Dini

Department of Chemistry

University of Rome La Sapienza

Piazzale Aldo Moro 5, Rome, 00185, Italy

Dr. Fabio Matteocci, Prof. Aldo Di Carlo

C.H.O.S.E - Centre for Hybrid and Organic Solar Energy, Department of Electronic Engineering University of Rome Tor Vergata

Rome, 00133, Italy

Prof. Aldo Di Carlo

L.A.S.E - Laboratory of Advanced Solar Energy

National University of Science and Technology NUST-MISiS

Russia

Felix Utama Kosasih, Dr. Giorgio Divitini, Prof. Caterina Ducati

Department of Materials Science and Metallurgy

University of Cambridge

27 Charles Babbage Road, Cambridge CB3 0FS, United Kingdom

Dr. Antonio Abate, Ganna Chistiakova, Dr. Lars Korte

Institute for Silicon Photovoltaics

Helmholtz-Zentrum Berlin für Materialien und Energie $\mathrm{GmbH}$

Kekuléstraße 5, D-12489 Berlin, Germany

mail: antonio.abate@helmholtz-berlin.de

Dr. Antonio Abate

State Key Laboratory of Photocatalysis on Energy and Environment Institute of Advanced Energy

Materials

Fuzhou University

Fuzhou, Fujian 350002, China

and

Department od Chemical, Materials and Production Engineering

University of Naples Federico II

Piazzale Tecchio 80, 80125 Fuorigrotta, Naples, Italy 


\section{WILEY-VCH}

S1.

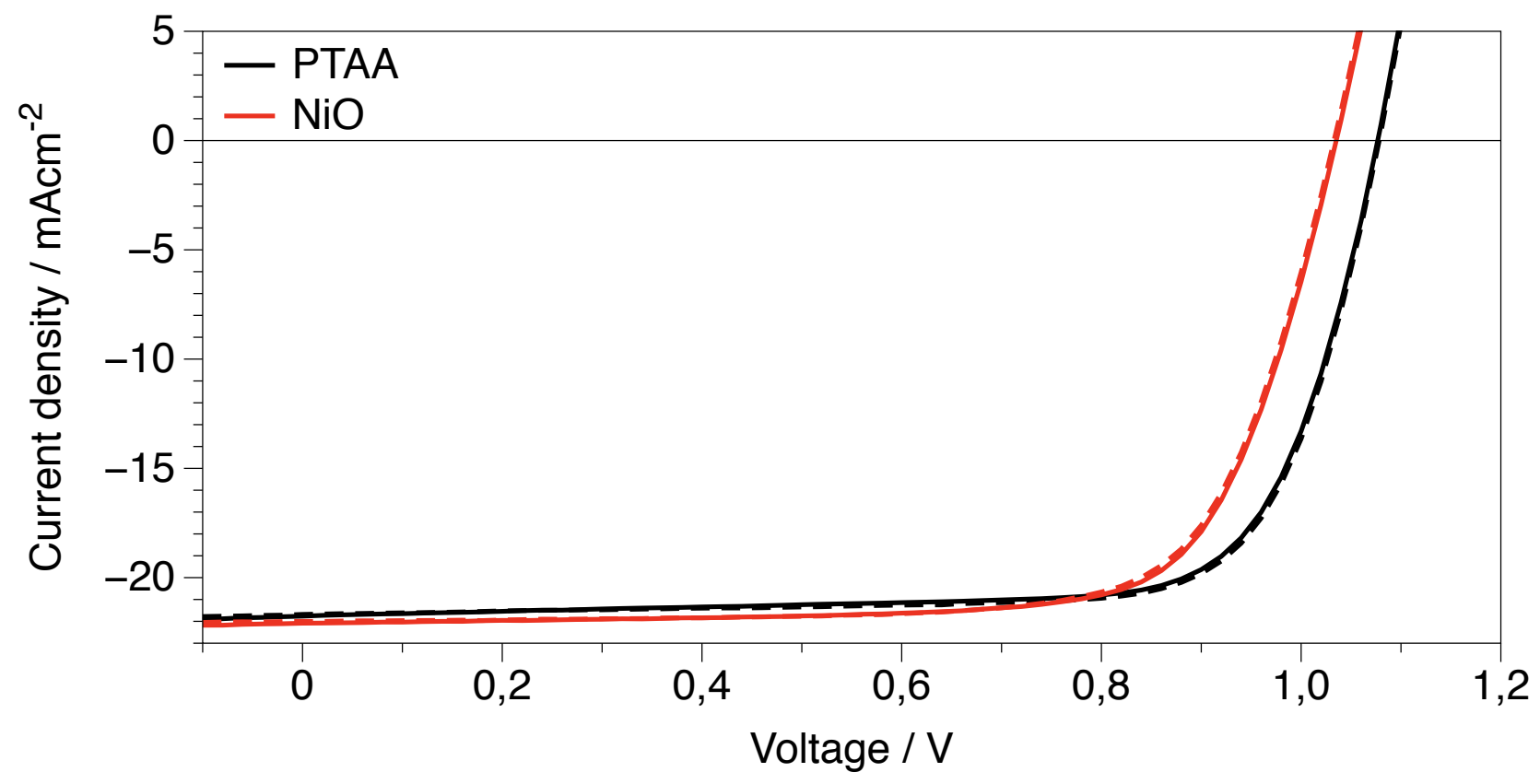

Representative JV curves under AM 1.5g illumination for PTAA and NiO based devices. The device employing PTAA benefits mostly from an higher Voc, due to lower interface recombination. PTAA devices deliver 17.8\% PCE $\left(\mathrm{Jsc}=21.7 \mathrm{mAcm}^{-2}, \mathrm{Voc}=1.08 \mathrm{~V}, \mathrm{FF}=0.76 \%\right)$

$\mathrm{NiO}$ devices deliver $16.8 \% \mathrm{PCE}\left(\mathrm{Jsc}=22.0 \mathrm{mAcm}^{-2}, \mathrm{Voc}=1.033 \mathrm{~V}, \mathrm{FF}=0.74 \%\right)$

Hysteresis is negligible for both HSL. 


\section{WILEY-VCH}

S2

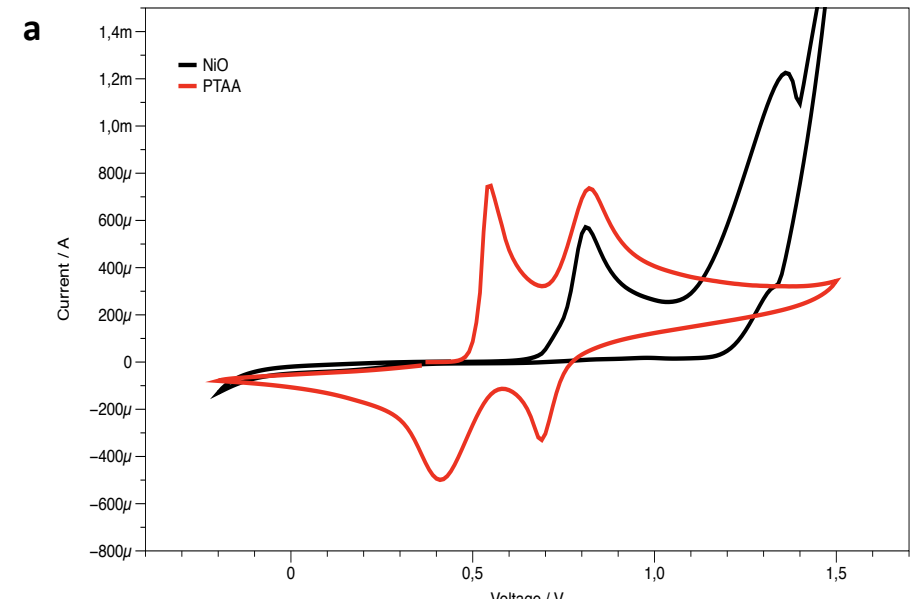

b

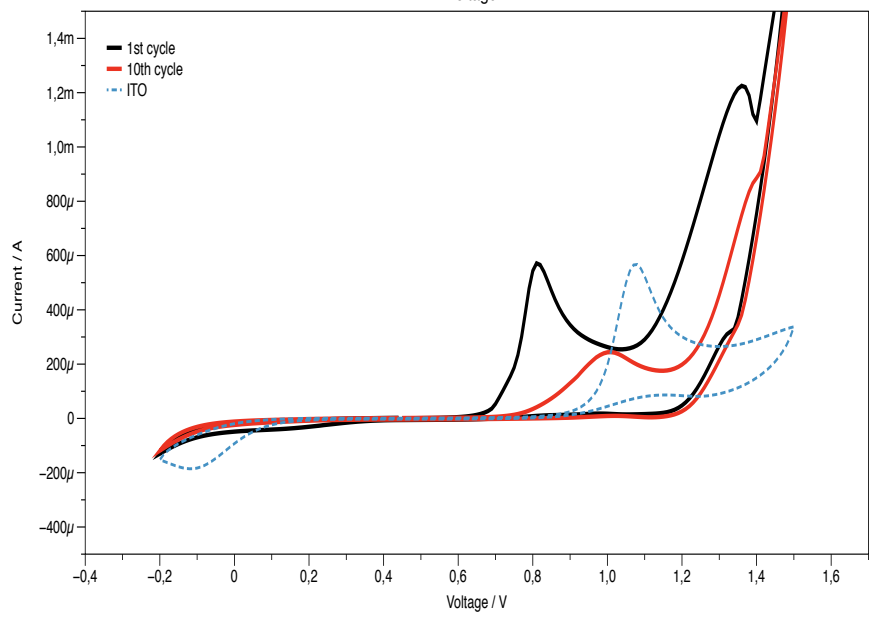

c

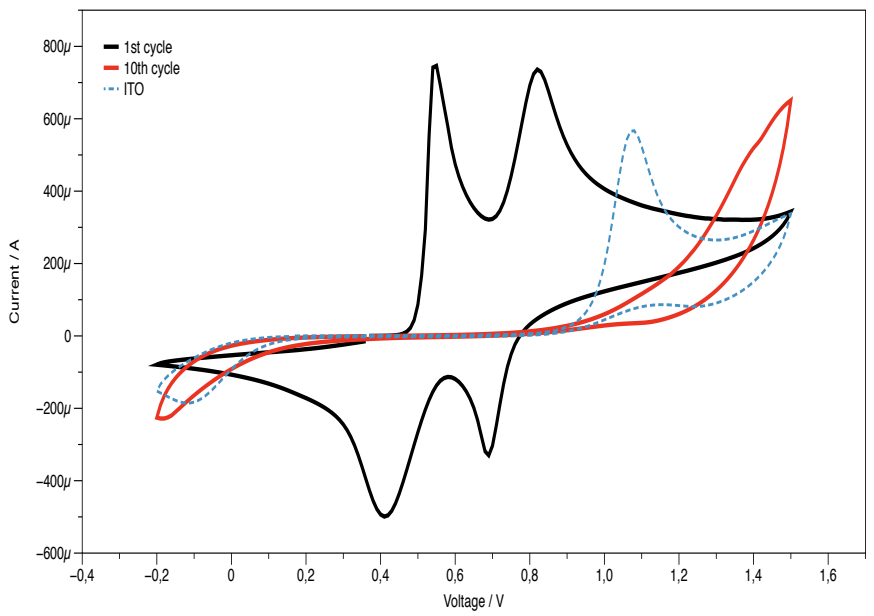

a) The cyclic voltammetry with $\mathrm{NiO}$ and PTAA on ITO as working electrode $(\mathrm{Ag} / \mathrm{AgCl}$ as $\mathrm{RE}$ and a $\mathrm{Pt}$ wire as $\mathrm{CE})$ and an aqueous electrolyte containing iodide $(0.1 \mathrm{M} \mathrm{KCl}$ and $5 \mathrm{mM} \mathrm{NaI})$. For both materials, the iodide oxidation occurs via two steps, shifted at higher voltages for $\mathrm{NiO}$. The key difference is the cathodic scan, where the reduction of iodine is observed for PTAA only. We recall 


\section{WILEY-VCH}

that there is no molecular iodine $\left(\mathrm{I}_{2}\right)$ in the electrolyte solution at the beginning of the experiment (while it develops during the experiments), therefore the reduction observed on PTAA must involve the iodine generated in the previous anodic scan. This suggests that after the anodic generation, the radical iodine remains bound to PTAA, available for following reduction. In b) and c) the comparison of the electrochemical stability of the two materials in these conditions. The NiO voltammogram stabilises after few cycles and the two peaks for the iodide oxidation repeatedly occur. The PTAA voltammogram is severely modified, with the entire loss of the iodide oxidation fingerprint, pointing to a severe chemical modification of PTAA during cyclic voltammetry. For comparison the voltammogram obtained on ITO is reported with the dashed line. 
WILEY-VCH

S3

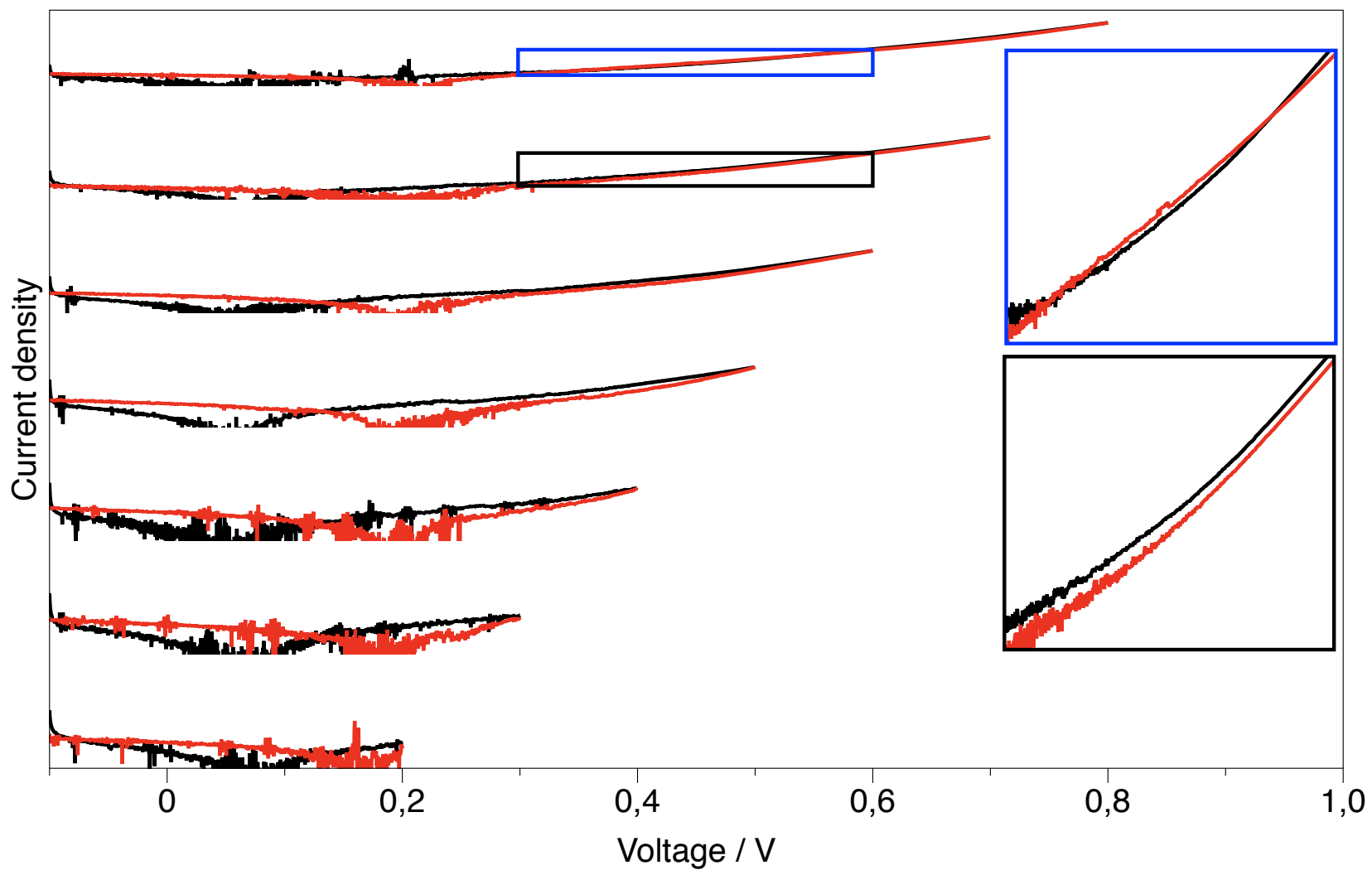

The complete experiment with the positive bias waveform with increasing amplitude. The forward scan is in black and the reverse scan is in red. In the two magnification it is possible to see that the loop is first detected when the voltage is scanned up to $0.8 \mathrm{~V}$ (blue square) and is not observed when cycling up to $0.7 \mathrm{~V}$ (black square). 


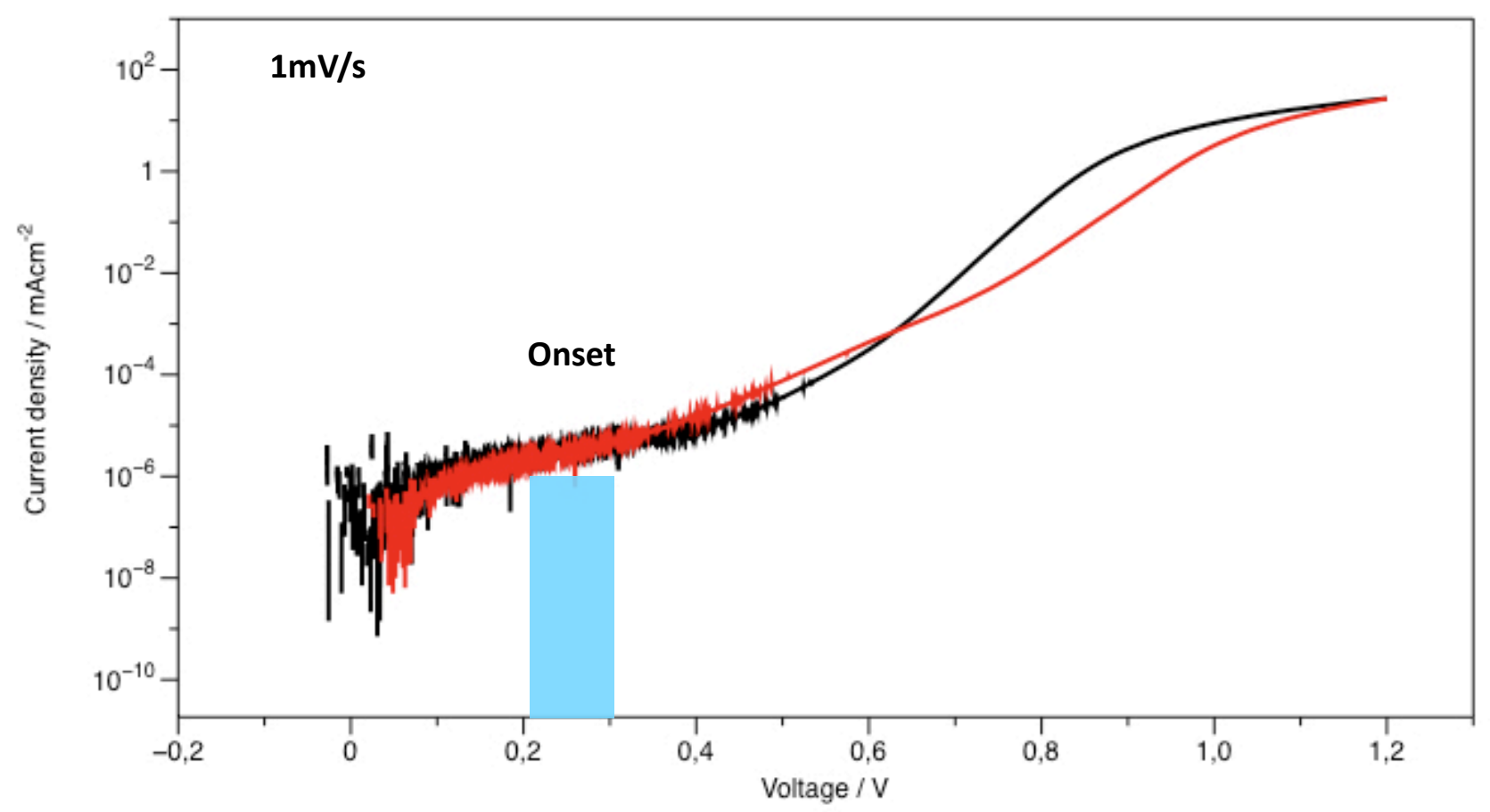

Dark JV recorded at $1 \mathrm{mV} / \mathrm{s}$ to have an accurate estimation on the onset for the potential dependent interaction of perovskite with $\mathrm{NiO}$, which is found to be between 0.2 and $0.3 \mathrm{~V}$. 


\section{WILEY-VCH}

a

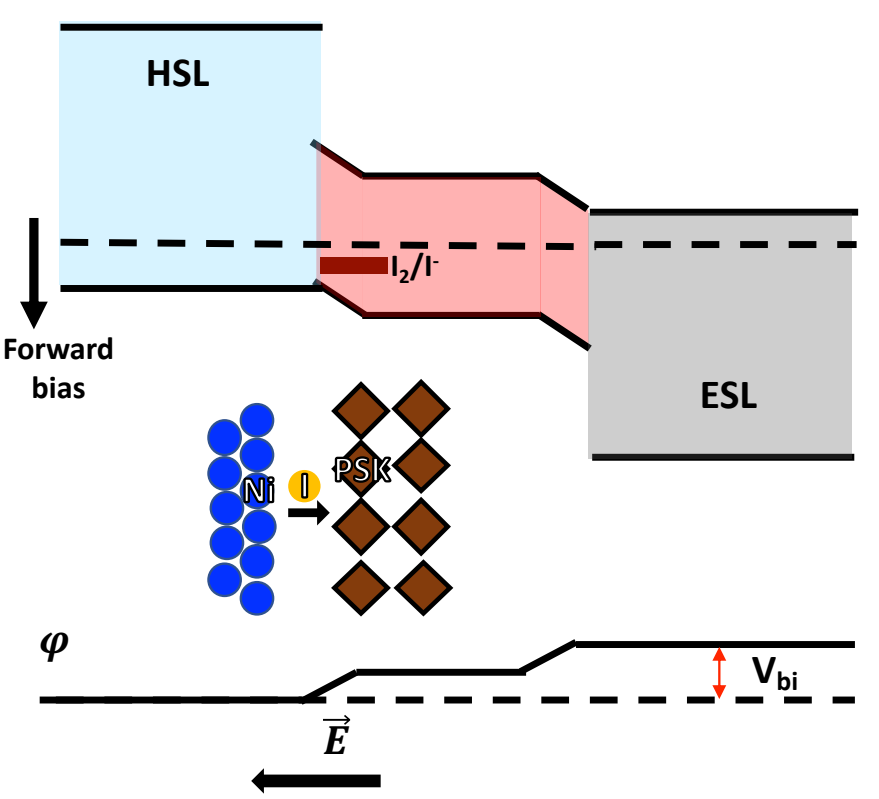

b

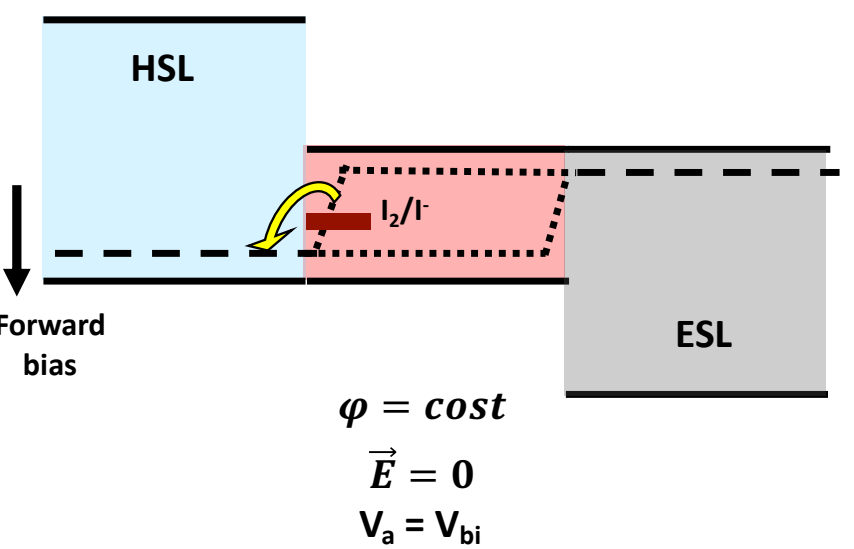

C
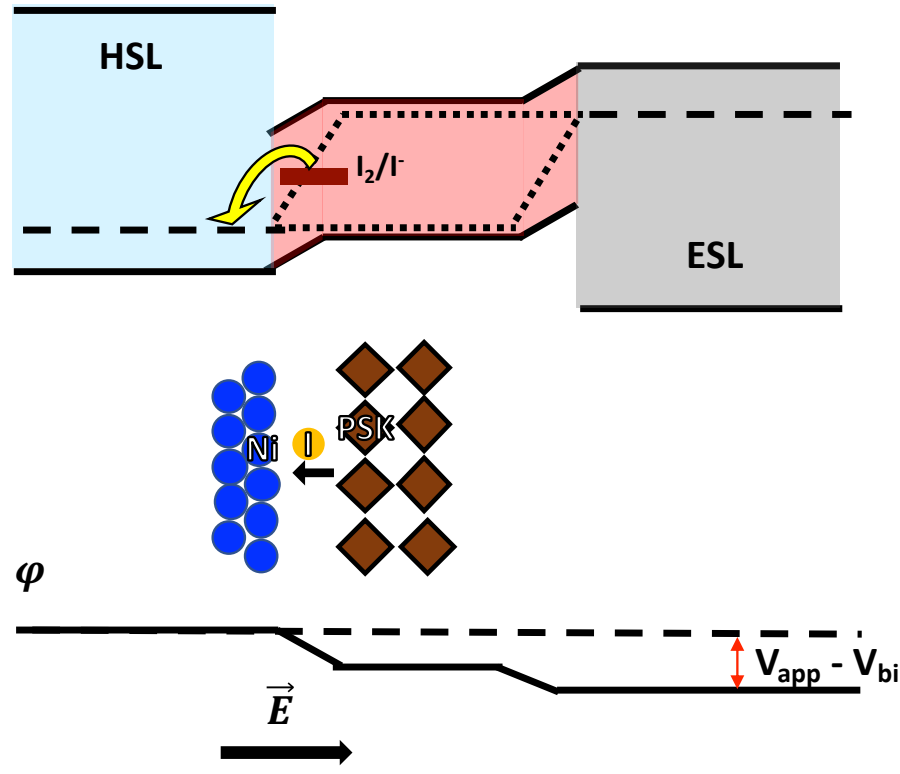


\section{WILEY-VCH}

Schematic sketches of the band line-up and band bending across a perovskite solar cell's absorber and carrier selective contacts. In these schemes we neglected the band bending relative to the selective contacts to simplify the picture. We stress that these are only graphical representation in line with what is referred as the "wiggle band model"(see ref [1]), i.e. band diagrams where the conduction and valence band of the perovskite absorber close to the interfaces are bent due to the ionic accumulation which screen the electric field in the bulk of the perovskite. Below the band diagrams we reported the profile of the electrical potential, $\varphi$.

Scheme a) represents the $\mathrm{p}-\mathrm{i}-\mathrm{n}$ junction experiencing the built-in potential $\left(\mathrm{V}_{\mathrm{bi}}\right)$. This is the state of the cell in the dark at $0 \mathrm{~V}$. As mentioned, the potential drops are localized at the two interfaces to the selective contacts. Due to these potential drops, i.e. the internal fields (gradients of the electric potentials), positively charged ions (defects) will accumulate at the HSL interface - in our case, the $\mathrm{NiO}$-, and the opposite at the ESL interface.

In b) we are applying an external bias equivalent to the built in potential. This brings about the split in the quasi-Fermi levels for electrons and holes in the perovskite layer, depicted with the dotted lines (note that depending on the interface recombination and the conductivity of the selective contacts, the splitting of the QFLs could also extend in the selective contacts). The $\mathrm{V}_{\mathrm{bi}}$ at the interfaces of the perovskite absorber depend on the differences between Fermi levels of selective contacts and the perovskite; the sum of both $\mathrm{V}_{\mathrm{biS}}$ (at the ETL and HTL interfaces) is usually between $0.8 \mathrm{~V}$ and $1.2 \mathrm{~V}$. When the applied voltage equals the built-in potential, the device experiences the relaxation of ionic distribution. Therefore, at $0.8 \mathrm{~V}$ a strong accumulation of iodide-based negatively charged defects (e.g. interstitial iodide) toward the $\mathrm{NiO}$ interface should not occur, while the hysteresis is already detectable (see figure 2c and S3) and this consideration suggests to disregard the ionic migration / accumulation as the only cause for the enhanced reactivity in the reverse scan.

In c) we show the band diagram when applying a forward bias larger than $\mathrm{V}_{\text {bi. }}$ In this case the accumulation of reactive iodide at the $\mathrm{NiO}$ interface is possible and, in our system, might enhance the interface reactivity. 


\section{WILEY-VCH}

We can now focus on the interface electrochemistry. In the band diagrams above we introduced the $\mathrm{I}_{2} / \mathrm{I}^{-}$redox couple inside the perovskite. To understand the reactivity at the $\mathrm{NiO}$ interface one has to consider the relative position of the $\mathrm{I}_{2} / \mathrm{I}^{-}$redox couple with respect to the Fermi level in $\mathrm{NiO}$.

In case a) the iodine reduction to iodide would be the spontaneous reaction. However, a negligible amount of iodine is expected to be in the perovskite and $\mathrm{NiO}$ does not enable the reduction (see fig S2). Therefore, the system is in equilibrium. With forward bias we move to the situation depicted in b) and c), where the Fermi level in $\mathrm{NiO}$ is below the redox potential of the $\mathrm{I}_{2} / \mathrm{I}^{-}$redox couple, allowing the iodide oxidation. In principle, this simple model holds for every hole selective contact. However, the non-capacitive hysteresis is the result of both the electrode activation and the relaxation process, and the back diffusion of oxidized iodine also plays a fundamental role in defining the JV loop. It is the kinetic interplay between these phenomena which defines the occurrence or not of the noncapacitive hysteresis, and those might differ markedly for different HSLs.

At last, we want to discuss the iodide-NiO interaction, i.e. the adsorption of iodide into the NiO film, and the effect of the interface electric field on it. This is sketched below the two band diagrams a), where the electric field hinders the absorption, and c), where the forward bias would drive a stronger interaction. Obviously, all the perovskite components will interact with the $\mathrm{NiO}$, but here we focus on the supposed electroactive iodide. We recall that a strong interaction between $\mathrm{NiO}$ and perovskite $\left(\mathrm{CH}_{3} \mathrm{NH}_{3} \mathrm{PbI}_{3}\right)$ has been modeled though computational simulation, where the formation of a Ni-I bond with high degree of covalence was observed[2]. Further, a similar equilibrium (except for the dependence on the electric field) has been recently proposed for the $\mathrm{TiO}_{2} / \mathrm{CH}_{3} \mathrm{NH}_{3} \mathrm{PbI}_{3}$ interface[3]. 

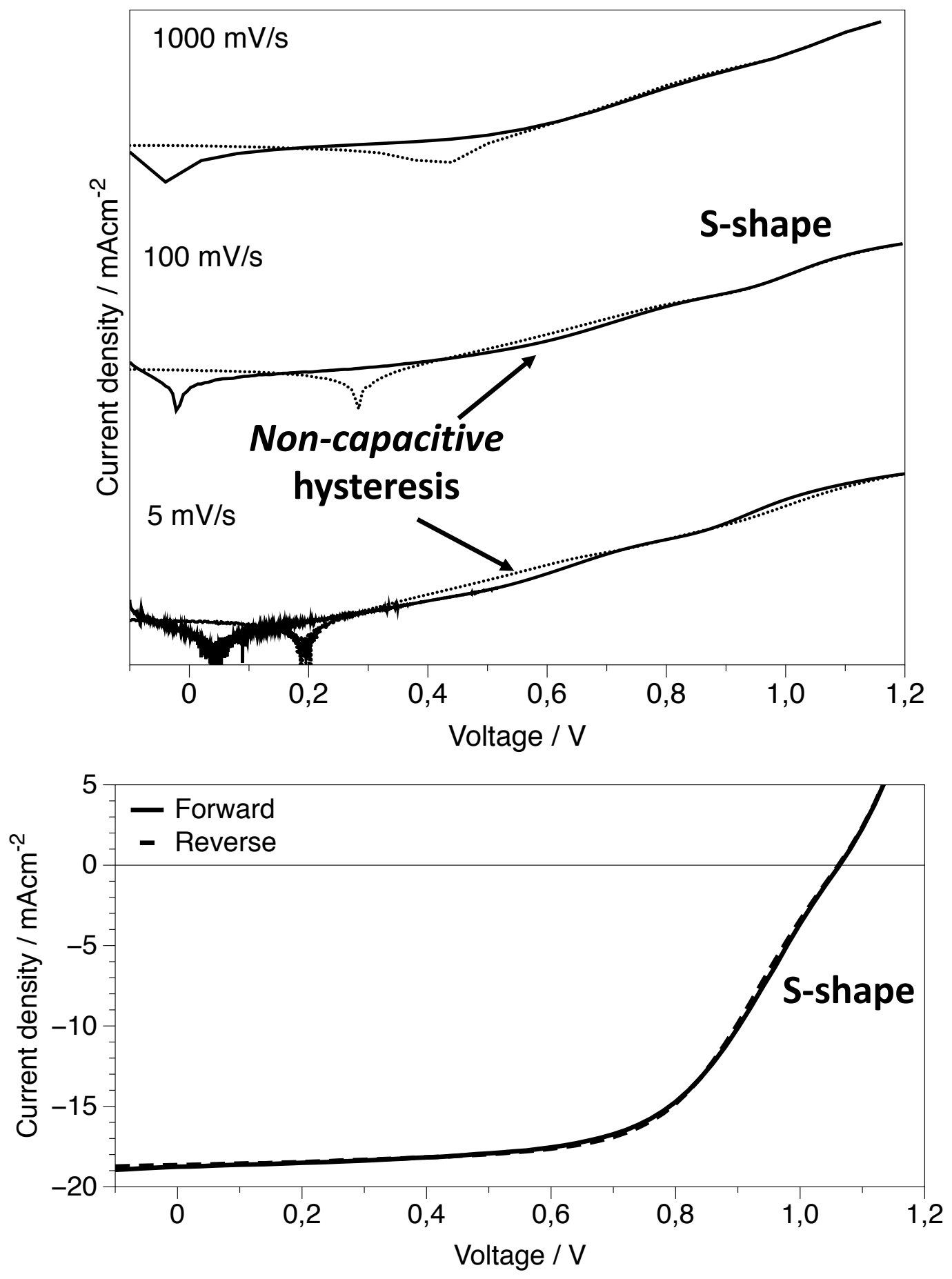

Reducing the thickness of PMMA (in this case deposited from a $0.8 \mathrm{mg} / \mathrm{mL}$ toluene solution) the efficiency of the device increase from $4 \%$ to $12 \%$. The increase in Jsc and FF directly correlate with a better hole extraction at the $\mathrm{NiO} / \mathrm{CsFAMA}$ interface. However, the device is less performing than reference one and from dark JV the non-capacitive hysteresis is found together with the occurrence of the typical kink referred as "S-Shape". 
WILEY-VCH

S7
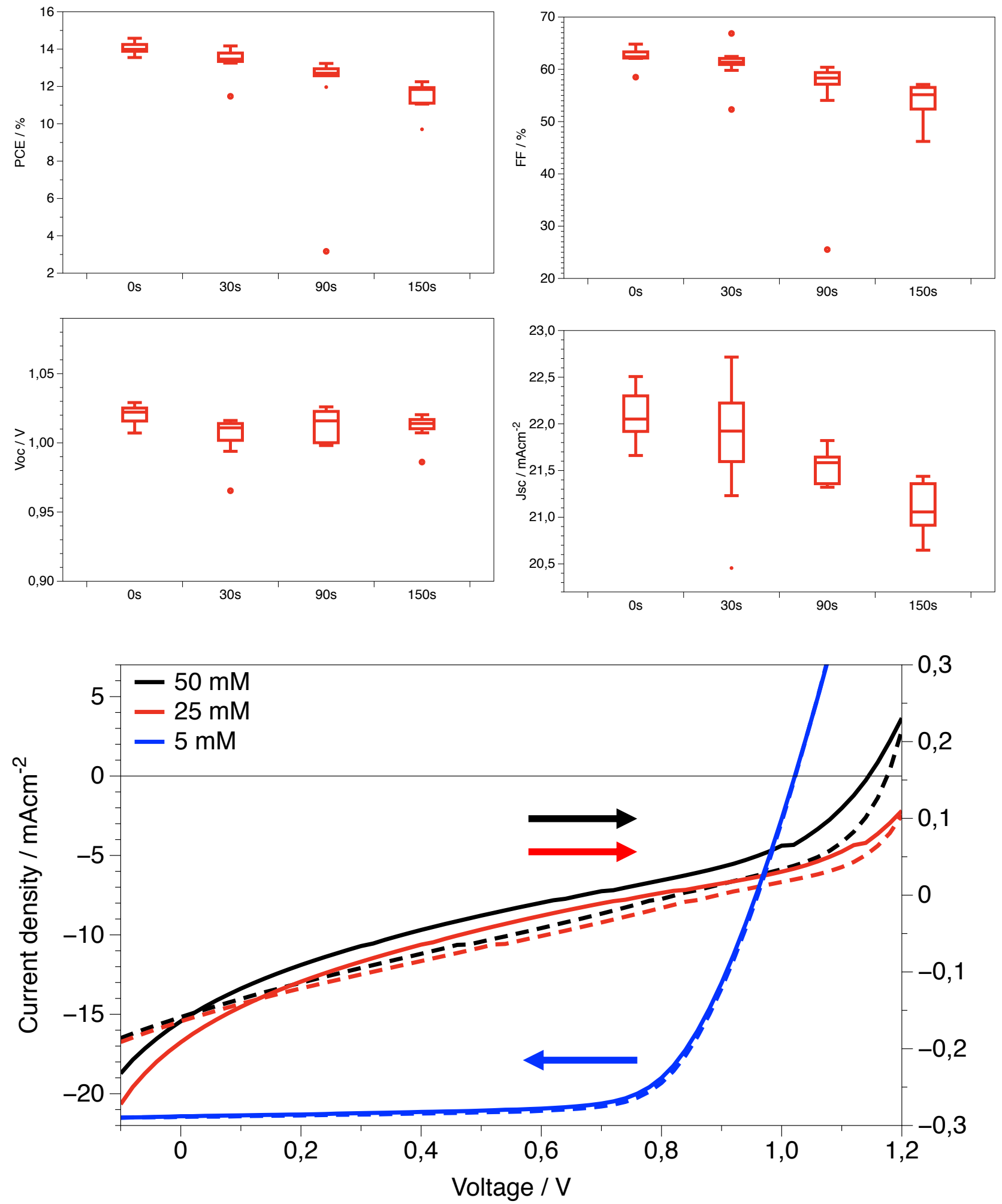

During the optimization of the device fabrication protocol, we evaluated the effect of UV-ozone treatment on $\mathrm{NiO}$. The $\mathrm{NiO}$ film resulted blackish even after short exposure to UV-ozone with an increasing loss in PV perfomances. Therefore, we avoided the UV treatment employed by Dagar et 


\section{WILEY-VCH}

al. [4] to cast the low temperature $\mathrm{MgO}$ on $\mathrm{NiO}$. This might be the reason for the organic ligands we observed through XPS. However, as in the case of $\mathrm{MgO}$, our layer acts as an insulator (see (S8)). In fact, by depositing the $\mathrm{MgO}(\mathrm{R})$ from a 25 or $50 \mathrm{mM} \mathrm{Mg}(\mathrm{AcO})_{2}$ ethanol solution the devices are completely insulated, with Jsc below $0.3 \mathrm{mAcm}^{2}$ and the PCE below $0.01 \%$. By decreasing the concentration to $5 \mathrm{mM}$ we recovered a good photovoltaic behaviour ( $\mathrm{PCE}>15 \%)$. Then, further optimizing to $3.5 \mathrm{mM}$, the reference device is outperformed and the non-capacitive hysteresis is suppressed, as discussed in the main text. 
WILEY-VCH

S8
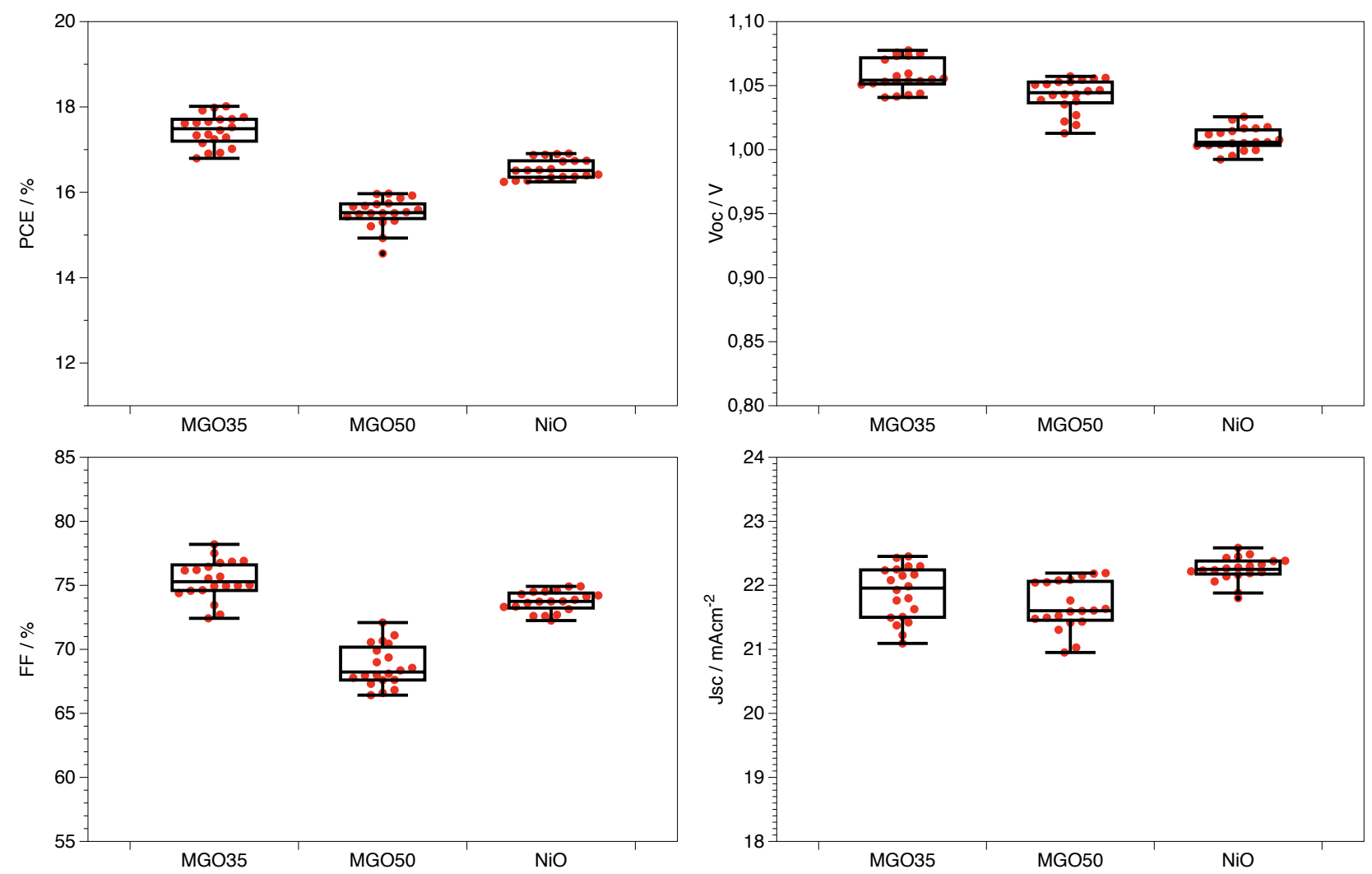

Box-chart of PV parameters from representative batch with $\mathrm{NiO}$ and $\mathrm{NiO} \mid \mathrm{MgO}(\mathrm{R})$ (from different concentration of the precursor solution, i.e. $3.5 \mathrm{mM}$ and $5 \mathrm{mM})$. 


\section{WILEY-VCH}

S9
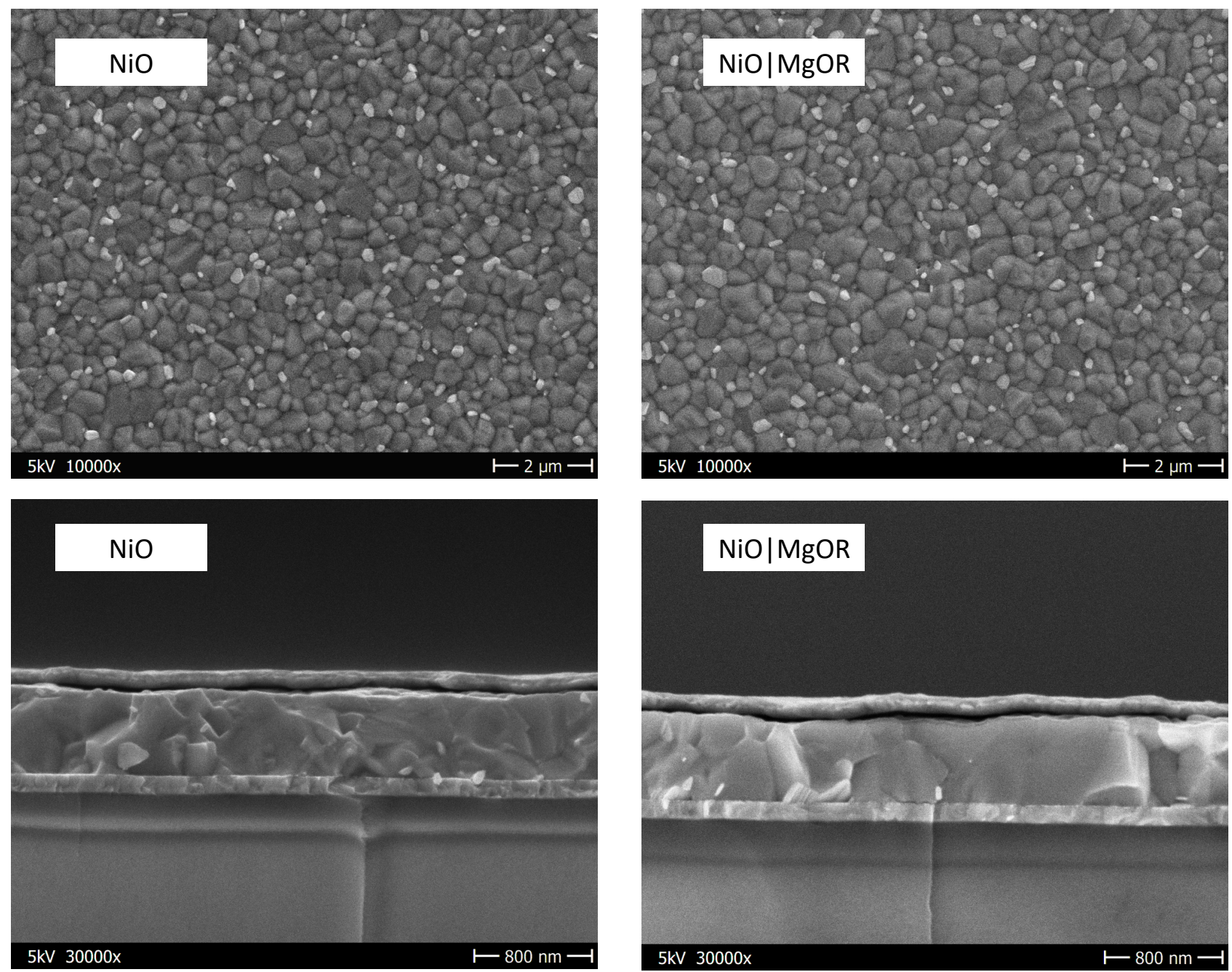

Top view and cross-section $\mathrm{SEM}$ of perovskite growth on $\mathrm{NiO}$ and on $\mathrm{NiO} / \mathrm{MgO}(\mathrm{R})$ layer. No difference is found, suggesting that the $\mathrm{MgO}(\mathrm{R})$ interlayer barely affect the perovskite growth. Similar conclusion is obtained from TEM analysis discussed in the main text. 


\section{WILEY-VCH}

S10

01s

a

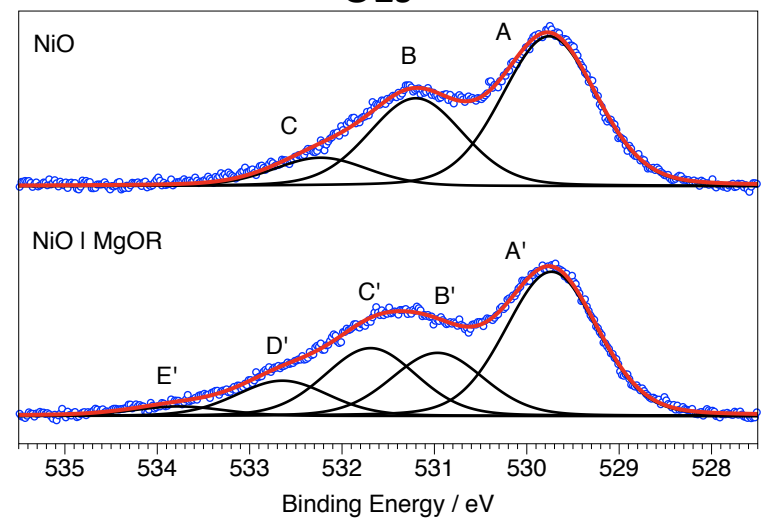

\section{C1s}

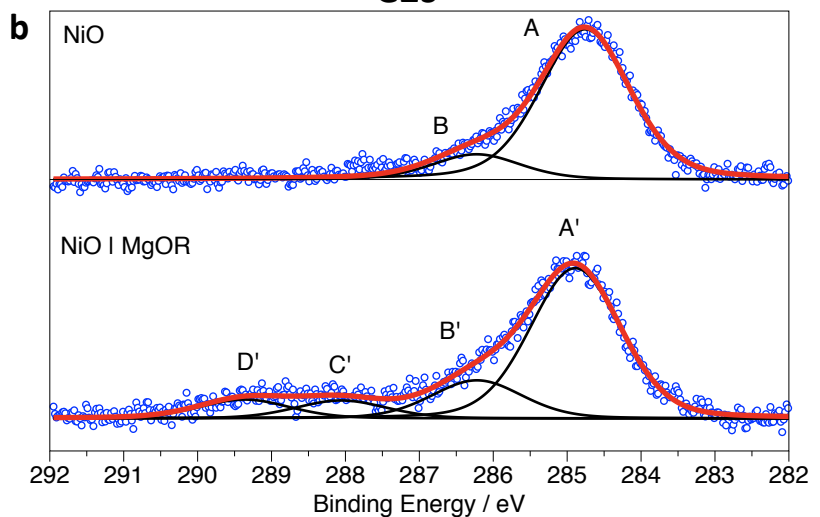

\begin{tabular}{|l|l|l|l|l|l|}
\hline $\begin{array}{l}\text { Component } \\
\text { O1s }\end{array}$ & NiO & Assignation & $\begin{array}{l}\text { Component } \\
\text { O1s }\end{array}$ & NiO | MgO(R) & Assignation \\
\hline A & $529.8 \mathrm{eV}$ & Lattice oxygen & A' $^{\prime}$ & $529.8 \mathrm{eV}$ & Lattice oxygen \\
\hline B & $531.2 \mathrm{eV}$ & Surface OH & B' $^{\prime}$ & $531.0 \mathrm{eV}$ & OH \\
\hline C & $532.3 \mathrm{eV}$ & Ads. oxygen & C' $^{\prime}$ & $531.7 \mathrm{eV}$ & O=C \\
\hline--- & --- & ---- & D' $^{\prime}$ & $532.6 \mathrm{eV}$ & O=C-O, C-O \\
\hline--- & ---- & ---- & E' $^{\prime}$ & $533.8 \mathrm{eV}$ & Ads. oxygen \\
\hline
\end{tabular}

\begin{tabular}{|l|l|l|l|l|l|}
\hline $\begin{array}{l}\text { Component } \\
\text { C1s }\end{array}$ & NiO & Assignation & $\begin{array}{l}\text { Component } \\
\text { C1s }\end{array}$ & NiO | MgO(R) & Assignation \\
\hline A & $284.8 \mathrm{eV}$ & Adv. carbon & A' $^{\prime}$ & $284.8 \mathrm{eV}$ & Adv. carbon \\
\hline B & $286.3 \mathrm{eV}$ & C-C, C-O (Adv. C) & B' & $286.2 \mathrm{eV}$ & C-C, C-O \\
\hline---- & ---- & ---- & C' $^{\prime}$ & $287.9 \mathrm{eV}$ & C=O \\
\hline---- & ---- & --- & D' & $289.2 \mathrm{eV}$ & O=C-O \\
\hline
\end{tabular}

The peak assignation is based on the relative scale of chemical shift reported, among the others, in $\operatorname{ref}[5],[6]$ and [7]. 


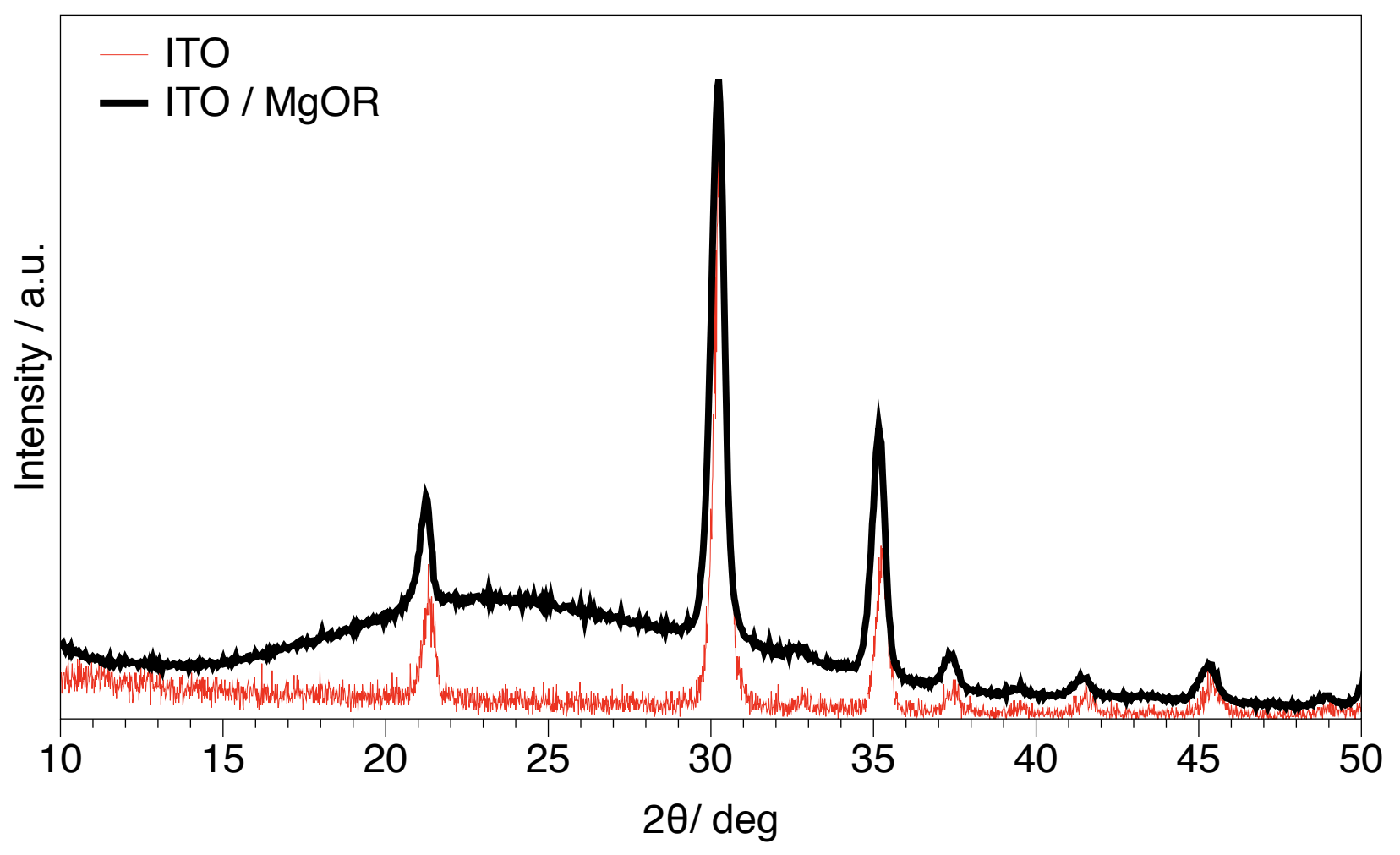

The XRD pattern of ITO/MgO(R) and ITO. To highlight eventual crystalline structure we deposited a thick layer of $\mathrm{MgO}(\mathrm{R})$ (from $50 \mathrm{mM}$ solution, 5 time spin). However, after the thermal treatment at $200^{\circ} \mathrm{C}$ only a broad peak typical of amorphous materials is found as difference in respect to the ITO alone. This states that the $\mathrm{MgO}(\mathrm{R})$ layer is amorphous. 


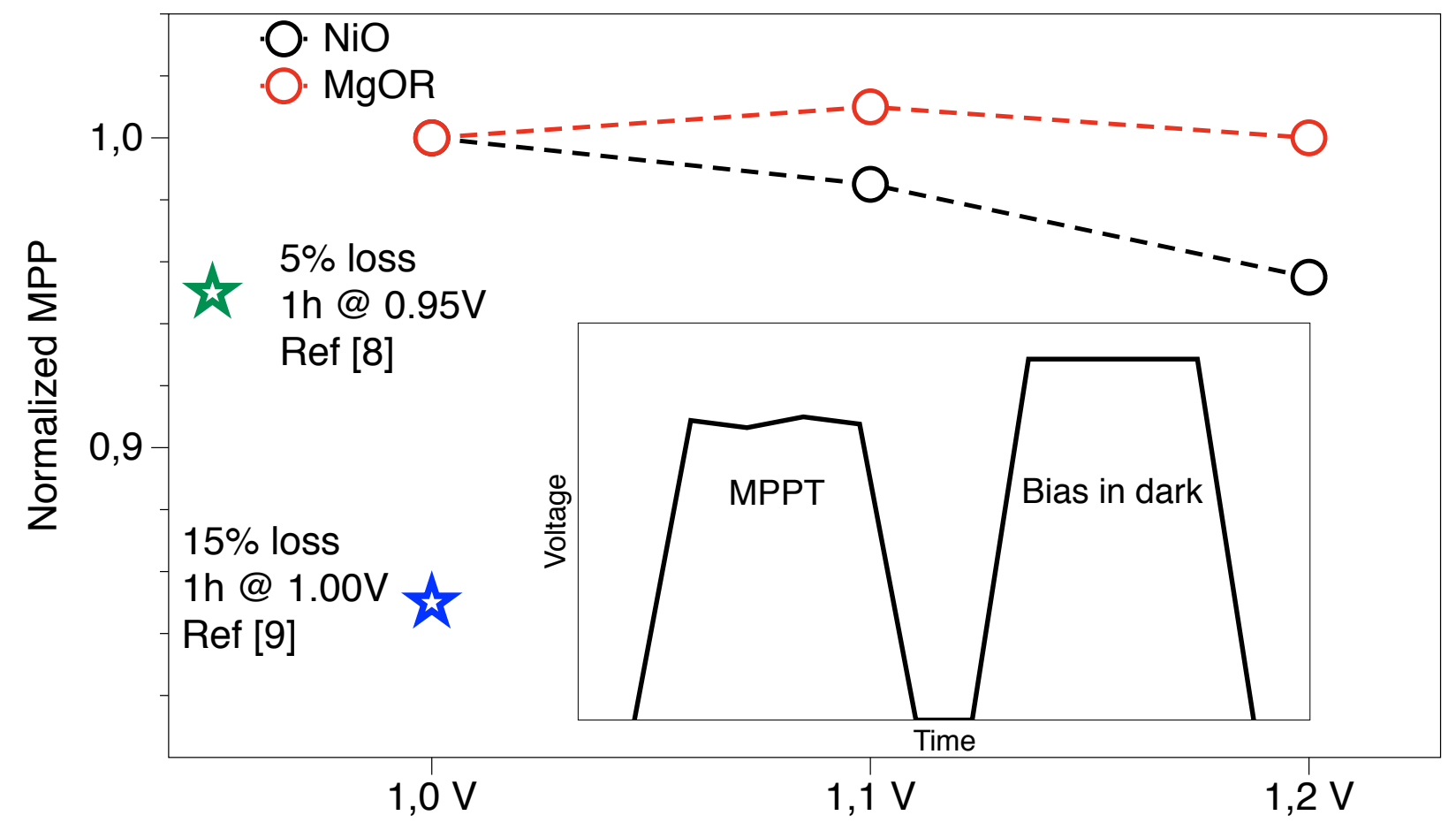

A comparison on the stability to forward bias in dark for device with and without $\mathrm{MgO}(\mathrm{R})$ interlayer. The efficiency is estimated with 10 minutes of MPPT before and after the electrical stress. At each potential value $(1.0 \mathrm{~V}, 1.1 \mathrm{~V}$ and $1.2 \mathrm{~V}) 3$ cycle of 20 minutes of forward bias in dark each were employed and the point in the graph represents the averaged results. The $\mathrm{MgO}(\mathrm{R})$ interlayer gives an higher stability in this test to the devices, with negligible loss during the whole experiment (loss around 5\% for the control).

The two stars reports the results of similar experiments reported in recent papers [8] and [9] on the effect of forward bias in dark on PSCs. 
a

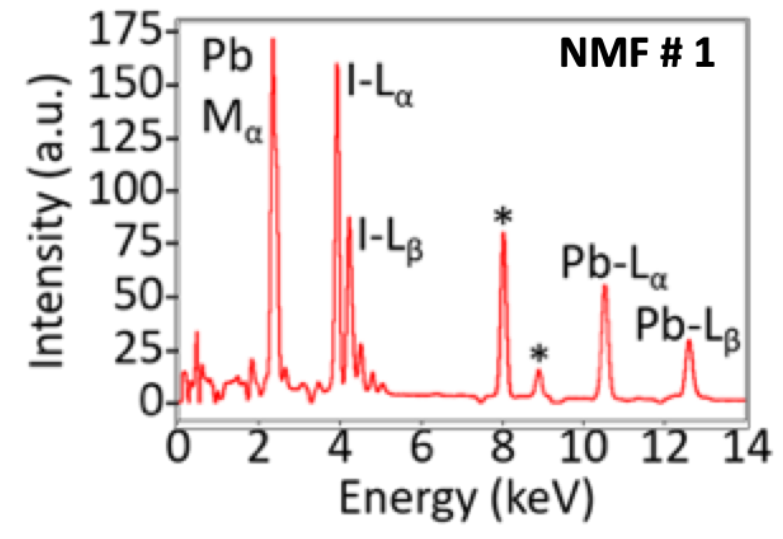

b

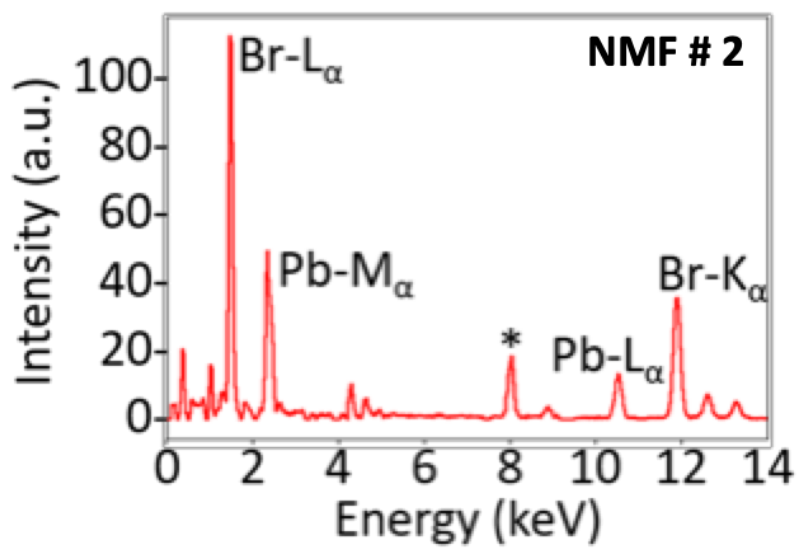

Spectral composition of NMF \#1 (a) and NMF \#2 (b).

$\mathrm{NMF} \# 1$ is mainly composed of $\mathrm{Pb}$ and I signals. NMF \#2 is composed of $\mathrm{Pb}$ and $\mathrm{Br}$ signal. These components are advantageous in describing the halide uniformity in the perovskite film 


\section{WILEY-VCH}

\section{Experimental}

Throughout the experiments (fabrication and measurement) the devices were never exposed to $\mathrm{O}_{2}$ or water to content in excess to $5 \mathrm{ppm}$ (most of time $<0.1 \mathrm{ppm}$ ). This shrewdness is intended to minimise the effect of water and oxygen in our investigation. For measurements performed in air we encapsulated the devices in glove box.

\section{NiOx deposition}

Patterned indium tin oxide (ITO) glass substrates $\left(25 \times 25 \mathrm{~mm}, 15 \Omega \mathrm{sq}^{-1}\right.$, patterned by Automatic Research $\mathrm{GmbH}$ ) were cleaned sequentially for 20 min with Mucasol 2\% solution in water (Schülke), Acetone, and Isopropanol in an ultrasonic bath at $40^{\circ} \mathrm{C}$. Before $\mathrm{NiOx}$ deposition, substrates were treated in an UV-ozone cleaner for $15 \mathrm{~min}$.

Nickel oxide is deposited from a $0.15 \mathrm{M} \mathrm{NiCl}_{2} 6 \mathrm{H}_{2} \mathrm{O}$ (Alfa Aesar) solution in anhydrous 2methoxyethanol (99.8\%, Sigma Aldrich) with the addition of $20 \mu \mathrm{L} / \mathrm{mL}$ of $\mathrm{HNO}_{3} 65 \%$ (Sigma Aldrich). After shaking the solution overnight at $60^{\circ} \mathrm{C}$ the spin-coating deposition of NiOx occurs via the following program: 500rpm (1s acceleration) for $1 \mathrm{~s}$ and $4000 \mathrm{rpm}$ ( $2 \mathrm{~s}$ acceleration) for $30 \mathrm{~s}$. After the spin-coating the substrate dry sequentially at $75^{\circ} \mathrm{C}$ for 10 minutes, at $120^{\circ} \mathrm{C}$ for 15 minutes and then is annealed at $300^{\circ} \mathrm{C}$ for 1 hour.

\section{$\operatorname{MgO}(\mathrm{R})$ and PMMA deposition}

$\mathrm{Mg}(\mathrm{AcO})_{2} 4 \mathrm{H}_{2} \mathrm{O}$ was dissolved in anhydrous ethanol to obtain a $50 \mathrm{mM}$ solution. Few minutes stirring at $50^{\circ} \mathrm{C}$ were enough to obtain a complete dissolution of the $\mathrm{Mg}$ precursor. It is important to note that this solution is not stable and leads to precipitation, probably following $\mathrm{Mg}^{2+}$ sol-gel chemistry in the slightly basic solution due to the acetate anion. The contact with moist air and longterm heating accelerate the precipitation, which is not reversible. The effect is less severe for more diluted solution ( $25 \mathrm{mM}, 5 \mathrm{mM}$ and $3.5 \mathrm{mM}$ employed in this work). The $\mathrm{Mg}(\mathrm{AcO})_{2} 4 \mathrm{H}_{2} \mathrm{O}$ solution was spinned on $\mathrm{NiO}$ at $4000 \mathrm{rpm}$ for $30 \mathrm{~s}$ in ambient atmosphere, dried at $75^{\circ} \mathrm{C}$ for 5 minutes and annealed at $200^{\circ} \mathrm{C}$ for 30 minutes.

PMMA was dissolved in toluene and heated at $60^{\circ} \mathrm{C}$ overnight before use it. The $\mathrm{Mg}(\mathrm{AcO})_{2} 4 \mathrm{H}_{2} \mathrm{O}$ solution was spinned on $\mathrm{NiO}$ at $4000 \mathrm{rpm}$ for $30 \mathrm{~s}$ in glove-box, then dried at $100^{\circ} \mathrm{C}$ for 10 minutes. 


\section{WILEY-VCH}

\section{Triple-cation Cs0.05(MA0.17FA0.83)0.95Pb(I0.83Br0.17)3 perovskite.}

First, $\mathrm{PbBr}_{2}(1.5 \mathrm{M})$ and $\mathrm{PbI}_{2}(1.5 \mathrm{M})$ were dissolved in a mixture of anhydrous DMF:DMSO (4:1 volume ratio) (Sigma Aldrich). After shaking overnight at $60^{\circ} \mathrm{C}$, the $\mathrm{PbI}_{2}$ and $\mathrm{PbBr}_{2}$ solutions were let cool down to room temperature and added to FAI and MABr powders respectively, to obtain $\mathrm{MAPbBr} 3$ and $\mathrm{FAPbI} 3$ solutions with a final concentration of $1.24 \mathrm{M}$ and a $9 \%$ excess of lead in both solutions. MAPbBr3 and FAPbI3 solutions were then mixed in a 17:83 volume ratio. Finally, the Caesium cation was added from a 1.5 M CsI solution in DMSO in a 5:95 volume ratio. The Perovskite solution was deposited on top of the HTM layer by spin-coating using the following program: 4000 $\operatorname{rpm}(5 \mathrm{~s}$ acceleration) for $35 \mathrm{~s}$ (total time $-40 \mathrm{~s}$ ). After $25 \mathrm{~s}, 600 \mu 1$ of Ethyl Acetate was dropped on the spinning substrate. After the spin-coating program, the perovskite-coated sample is annealed at $100^{\circ} \mathrm{C}$ for $60 \mathrm{~min}$ on a hotplate.

\section{Electron selective contact and metal electrode}

On top of the perovskite, $23 \mathrm{~nm}$ of $\mathrm{C} 60$ and $8 \mathrm{~nm}$ of BCP were deposited by thermal evaporation (Mbraun ProVap 3G) with evaporation rates below $0.2 \AA / \mathrm{s}$ at a pressure of under $1 \mathrm{E}-6 \mathrm{mbar}$. At the end, $100 \mathrm{~nm}$ of $\mathrm{Cu}$ was evaporated at a rate of $0.08-1.2 \AA / \mathrm{s}$. The active area is defined by the overlap of patterned ITO and the metal electrode, which is $0.16 \mathrm{~cm} 2$.

\section{Device and Materials characterization}

Current-voltage $(\mathrm{J}-\mathrm{V})$ characteristics under 1 sun equivalent illumination were recorded using a Wavelabs Sinus-70 LED class AAA in air, calibrated with a Silicon reference solar cell from Fraunhofer ISE. J-V scans were performed with a Keithley 2400 SMU, controlled by a measurement control program written in LabView. The voltage values are swept in $20 \mathrm{mV}$ steps with variable integration time and settling time after voltage application to control the scan rate.

Transient PhotoVoltage (TPV) and Charge Extraction (CE) were performed with a commercial setup for advanced photo-electrical characterization (Arkeo from Cicci Research srl) based on an high speed Waveform Generator that drives an high speed LED (5000 Kelvin). The device is connected to a transimpedance amplifier and a differential voltage amplifier to monitor short circuit current or open circuit voltage. The light intensity can be varied between 0.1 and 2 equivalent Sun. 


\section{WILEY-VCH}

Electrochemical investigation were carried out employing a 3 electrode electrochemical cell with $\mathrm{Ag} / \mathrm{AgCl}$ reference electrode and a platinum wire as counter electrode. The organic electrolyte was composed of $\mathrm{NaI}(5 \mathrm{mM})$ and $\mathrm{TBABF} 4(0.1 \mathrm{M})$ in acetonitrile $(\mathrm{ACN})$. The aqueous electrolyte was composed of $\mathrm{NaI}(5 \mathrm{mM})$ and $\mathrm{KCl}(0.1 \mathrm{M})$. We employed a Ivium CompactStat potentiostat equipped with IviumSoft software. The same potentiostat was employed for the analysis of the evolution of the hysteresis with repeated potential cycling (figure $2 \mathrm{~d}, 2 \mathrm{e}$ and $2 \mathrm{f}$ ).

SEM images were recorded using a Hitachi S4100 or ZEISS Auriga 60 at $5 \mathrm{kV}$ acceleration voltage and $10 \mathrm{k} \times$ and $30 \mathrm{k} \times$ magnification.

The grazing incident X-ray diffraction for thin film analysis (GIXRD) measurements was conducted with Bruker D8 with energy-dispersive Sol-X detector using Cu K-alpha source. The scan range is from 20 to 70 degree with 0.5 degree incident angle and step size is 0.02 degree. All of samples were measured in ambient condition

XPS measurements were conducted employing $\mathrm{Mg} \mathrm{K \alpha}$ excitation and using ScientaOmicron Argus CU electron analyzer. After the deposition samples were transferred via glovebox without air exposure. Peak fitting was performed with free software Fityk. We subtracted a Shirley background and then employed pseudo-Voigt function for peak fit.

For Scanning transmission electron microscopy (STEM) characterisation cross-sectional lamellae of the devices were prepared by focused ion beam (FIB, FEI Helios Nanolab) using a standard approach with minimal exposure to air between sample preparation and STEM characterisation. STEM-HAADF imaging and STEM-EDX spectroscopy were carried out in a FEI Tecnai Osiris operated at $200 \mathrm{kV}$ and equipped with a Bruker Super-X EDX silicon drift detector with a collection solid angle of $\sim 0.9$ sr. Elemental maps were acquired with a probe current of $\sim 0.25 \mathrm{nA}$, spatial sampling of $10 \mathrm{~nm} /$ pixel, and dwell time of $50 \mathrm{~ms} /$ pixel. Data were acquired with Tecnai Imaging and Analysis (TIA) and analysed with HyperSpy.[10] 


\section{WILEY-VCH}

\section{References}

[1] L. Bertoluzzi, R. A. Belisle, K. A. Bush, M. D. Mcgehee, B. C. O. Regan, 2018, DOI 10.1021/jacs.8b04405.

[2] W. Nie, H. Tsai, J. C. Blancon, F. Liu, C. C. Stoumpos, B. Traore, M. Kepenekian, O. Durand, C. Katan, S. Tretiak, J. Crochet, P. M. Ajayan, M. Kanatzidis, J. Even, A. D. Mohite, Adv. Mater. 2018, 30, 1.

[3] R. A. Kerner, B. P. Rand, J. Phys. Chem. Lett. 2017, 8, 2298.

[4] J. Dagar, S. Castro-Hermosa, G. Lucarelli, F. Cacialli, T. M. Brown, Nano Energy 2018, 49, 290.

[5] T. I. T. Okpalugo, P. Papakonstantinou, H. Murphy, J. McLaughlin, N. M. D. Brown, Carbon N. Y. 2005, 43, 153.

[6] C. M. Chen, J. Q. Huang, Q. Zhang, W. Z. Gong, Q. H. Yang, M. Z. Wang, Y. G. Yang, Carbon N. Y. 2012, 50, 659.

[7] G. P. López, D. G. Castner, B. D. Ratner, Surf. Interface Anal. 1991, 17, 267.

[8] J. Barbé, V. Kumar, M. J. Newman, H. K. H. Lee, S. M. Jain, H. Chen, C. Charbonneau, C. Rodenburg, W. C. Tsoi, Sustain. Energy Fuels 2018, 2, 905.

[9] H. J. Jung, D. Kim, S. Kim, J. Park, V. P. Dravid, B. Shin, Adv. Mater. 2018, 1802769, 1802769.

[10] S. Cacovich, F. Matteocci, M. Abdi-Jalebi, S. D. Stranks, A. Di Carlo, C. Ducati, G. Divitini, ACS Appl. Energy Mater. 2018, 1, 7174. 